\title{
DAMEfinder: a method to detect differential allele-specific methylation
}

\author{
Stephany Orjuela ${ }^{1,2}$, Dania Machlab ${ }^{3}$, Mirco Menigatti ${ }^{2}$, Giancarlo Marra ${ }^{2}$ and Mark D. Robinson ${ }^{1 *}$ (0)
}

\begin{abstract}
Background: DNA methylation is a highly studied epigenetic signature that is associated with regulation of gene expression, whereby genes with high levels of promoter methylation are generally repressed. Genomic imprinting occurs when one of the parental alleles is methylated, i.e., when there is inherited allele-specific methylation (ASM). A special case of imprinting occurs during $X$ chromosome inactivation in females, where one of the two X chromosomes is silenced, to achieve dosage compensation between the sexes. Another more widespread form of ASM is sequence dependent (SD-ASM), where ASM is linked to a nearby heterozygous single nucleotide polymorphism (SNP).
\end{abstract}

Results: We developed a method to screen for genomic regions that exhibit loss or gain of ASM in samples from two conditions (treatments, diseases, etc.). The method relies on the availability of bisulfite sequencing data from multiple samples of the two conditions. We leverage other established computational methods to screen for these regions within a new R package called DAMEfinder. It calculates an ASM score for all CpG sites or pairs in the genome of each sample, and then quantifies the change in ASM between conditions. It then clusters nearby CpG sites with consistent change into regions. In the absence of SNP information, our method relies only on reads to quantify ASM. This novel ASM score compares favorably to current methods that also screen for ASM. Not only does it easily discern between imprinted and non-imprinted regions, but also females from males based on X chromosome inactivation. We also applied DAMEfinder to a colorectal cancer dataset and observed that colorectal cancer subtypes are distinguishable according to their ASM signature. We also re-discover known cases of loss of imprinting.

Conclusion: We have designed DAMEfinder to detect regions of differential ASM (DAMEs), which is a more refined definition of differential methylation, and can therefore help in breaking down the complexity of DNA methylation and its influence in development and disease.

Keywords: DNA methylation, Differential methylation, Allele-specific methylation (ASM), Imprinting

\section{Background}

Epigenetic modifications refer to mitotically heritable, chemical variations in DNA and chromatin in the absence of changes in the DNA nucleotide sequence itself $[1,2]$. Although there are a large number of such

\footnotetext{
*Correspondence: mark.robinson@imls.uzh.ch

${ }^{1}$ Institute of Molecular Life Sciences and SIB Swiss Institute

of Bioinformatics, University of Zurich, Winterthurerstrasse 190, 8057 Zurich, Switzerland

Full list of author information is available at the end of the article
}

documented phenomena, DNA methylation (i.e., methyl groups added to cytosines in mammalian DNA, mostly in CpG dinucleotides) stands out because the mechanism of heritability, via maintenance methyltransferases, is welldetermined [3-5]. In addition, due to well-known effects of chemical reactions, such as sodium bisulfite conversion of cytosines to uracils [6], and biochemical reactions like TET-pyridine borane conversion of 5-methylcytosine to dihydrouracil [7], the interrogation of DNA methylation level across the genome can be sampled and quantified at each cytosine.

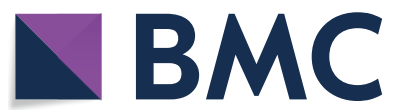

(c) The Author(s) 2020. This article is licensed under a Creative Commons Attribution 4.0 International License, which permits use, sharing, adaptation, distribution and reproduction in any medium or format, as long as you give appropriate credit to the original author(s) and the source, provide a link to the Creative Commons licence, and indicate if changes were made. The images or other third party material in this article are included in the article's Creative Commons licence, unless indicated otherwise in a credit line to the material. If material is not included in the article's Creative Commons licence and your intended use is not permitted by statutory regulation or exceeds the permitted use, you will need to obtain permission directly from the copyright holder. To view a copy of this licence, visit http://creativeco mmons.org/licenses/by/4.0/. The Creative Commons Public Domain Dedication waiver (http://creativecommons.org/publicdomain/ zero/1.0/) applies to the data made available in this article, unless otherwise stated in a credit line to the data. 
DNA methylation plays a role in several biological phenomena. It is believed to be associated with gene expression, with the canonical relationship suggesting that transcriptional units with high levels of promoter methylation are repressed or silenced, although not all genes with unmethylated promoters are switched on, since other epigenetic mechanisms of silencing may come into play [8].

Genomic imprinting, where genes are expressed in a parent-of-origin manner [9], is also regulated by DNA methylation. Imprinting occurs via allele-specific methylation (ASM), in which only the paternal or the maternal allele is methylated in all or most of the tissues of an individual [9]. This methylation asymmetry is conferred during gametogenesis in the parental germlines, or during early embryogenesis after fertilization, and will remain during the lifetime of the offspring [10]. A recent survey [11] reported 228 genes linked to imprinted control, and from those, 115 linked to imprinted regulation in human placenta. These genes are known for their roles in embryonic and fetal development, placental formation, cell growth and differentiation, metabolism and circadian clock regulation [11]. In fact, loss of imprinting and abnormal expression of imprinted genes are implicated in severe congenital diseases, like the neurodevelopment disorders Angelman and Prader-Willi syndromes. The first is caused by the lack of maternal $U B E 3 A$ gene expression, and the second by loss of paternal expression of several contiguous genes on chromosome 15q11-q13 [12]. Furthermore, disruption of imprinting in somatic cells has been implicated in the pathogenesis of different cancers, like loss of imprinting within the H19/IGF2 imprinting control region in colorectal cancer [13], and gain of imprinting at $11 \mathrm{p} 15$ in hepatocellular carcinoma [14].

A special and well-characterized case of imprinting occurs during $\mathrm{X}$ chromosome inactivation $(\mathrm{XCI})$, where one of the two $\mathrm{X}$ chromosomes is randomly silenced via DNA methylation and other epigenetic mechanisms, early in development in each cell of a female, to achieve dosage compensation between the sexes [15].

Beside imprinting and $\mathrm{XCI}$, the rest of the genome is thought to be symmetrically methylated across both alleles. However, sequence-dependent ASM (SD-ASM) has been frequently reported in the last 10 years and appears to be widespread in the human genome [16-21]. In this case, the DNA methylation asymmetry between the parental alleles appears to be causally related to the presence of a single nucleotide polymorphism (SNP). As for imprinted ASM and XCI, SD-ASM can be associated with silencing of one of the two parental gene copies, likely mediated by cis-acting, allele-specific changes in affinity of DNA-binding proteins [21]. SD-ASM appears to be tissue-specific [22, 23], thus it is commonly believed that the interaction between genetic variants (i.e., SNPs) and epigenetic mechanisms (i.e., effects of DNA methylation asymmetry on gene expression) modulate the susceptibility of the general population to frequent, multi-factorial diseases affecting specific organs. An example of this is SD-ASM in the PEAR1 intron 1, which is linked to platelet reactivity and cardiovascular disease [24]. Another example is SD-ASM in FKBP5, a gene encoding a cochaperone of the glucocorticoid receptor with a potential role in the stress hormone-regulating hypothalamic-pituitary-adrenal axis [25], which poses an increased risk to stress-related psychiatric disorders in individuals who suffered an abuse during childhood [26]. Although the modulation of the susceptibility to a complex disease by SD-ASM is generally weak and influenced by environmental factors, it is worth noting that $5-10 \%$ of all SNPs might be associated with SD-ASMs in the genome of a given tissue of a given individual [19, 20,27].

Although there are several technologies to study DNA methylation, such as microarrays that genotype bisulfiteconverted DNA, or lower resolution capture technologies such as methyl-binding domain (MBD) sequencing [28], or methylated DNA immunoprecipitation (MeDIP) sequencing [29], bisulfite sequencing (BS-seq) remains distinct for the ability to read out DNA methylation of a single allele at base-resolution. Importantly, BS-seq can be conducted both in an unbiased genome-wide fashion, or in combination with technologies that focus the sequencing to particular regions, either by making use of hybridization or by enzyme digestions [30].

Recent studies have obtained ASM readouts from mapped bisulfite reads, by assigning them to the alleles of each known heterozygous SNP. Methylation levels are then determined for all allele-linked cytosines in the reads (see $[20,31,32]$ for recent examples). The ASM calculated in this way is interpreted as SD-ASM, and it does not include imprinted ASM nor XCI, since they are not necessarily sequence dependent. Calculating ASM in this fashion is limited by the availability of SNP information from either DNA-seq or SNP-array data, or directly from the BS-seq reads [33]. However, performing different types of high-throughput experiments is economically restrictive and time consuming, and deriving SNPs from $\mathrm{BS}$-seq reads can be problematic due to bisulfite conversion of DNA (i.e., distinguishing a C/T SNP from a C/T conversion of a methylated cytosine) and imbalanced strand coverage (i.e., when the Watson and Crick strands are not equally or highly covered) [33].

Considering these limitations in ASM detection, a couple of studies have sought to make sole use of BS-seq reads to screen for the full spectrum of ASM. The tools allelicmeth and amrfinder (from the same authors) [34] 
are the only available executable methods that detect ASM without SNP information. In brief, the allelicmeth method creates a contingency table with the counts of methylated and unmethylated reads covering a pair of CpG sites. A score is calculated via Fisher's exact test that represents the probability that both CpG sites have an equal proportion of methylated-unmethylated reads. amrfinder also calculates ASM but at a regional level. It fits two statistical models, one assuming that both alleles are equally methylated, and the other assuming different methylation states for the two alleles. A region is considered to have ASM by comparing the likelihoods of the two models. A more recent algorithm termed MethylMosaic relies on the principle that bimodal methylation patterns, independent from the genotype, are a good indicator of ASM [35]; however, to our knowledge there is no publicly available implementation.

Based on the current state of ASM detection from BSseq reads, we set out to develop a simple yet effective method to screen for genomic regions that exhibit loss or gain of ASM between samples from distinct conditions. The methods mentioned above detect ASM in individual samples; however, they do not allow a flexible comparison between groups of samples, such as that performed in a typical differential methylation analysis [36, 37], where the goal is to find the effect of treatments or diseases on methylation, reflected as increase or decrease of methylation levels. Here, we are interested in performing such differential analysis but focusing on the effect of ASM, reflected as gain or loss of allele specificity. For this task, we introduce DAMEfinder (Differential Allele-specific MEthylation finder), an $\mathbf{R}$ package [38] that consists of (i) a scoring function that reflects ASM for several samples; (ii) integration with limma [39] and bumphunter [40] to detect differentially allele-specific methylated regions (DAMEs); and (iii) accurate estimation of false discovery rates (FDR). We demonstrate the ASM score and DAMEfinder on two real datasets, one based on targeted enrichment BS-seq, comparing normal colonic mucosa to cancerous colorectal lesions, and another on whole genome BS-seq (WGBS), comparing blood monocytes from healthy females and males.

\section{Results}

\section{The overall DAMEfinder workflow}

Figure 1 gives an overview of the pipeline. We make considerable use of existing tools and keep inputs/outputs in standard formats. To make use of the package, the user must independently use bismark to map paired-end BSseq reads against a reference genome (Fig. 1a). Once this is done, the user has the option to detect ASM for each sample in two ways: (1) using the output from methtuple [41], which computes read counts of pairs of nearby CpG sites. From these counts, we compute an ASM score; (2) using an additional VCF file containing heterozygous SNPs. For each SNP we call methylation from the reads containing that SNP, and calculate an ASM score for each CpG site (Fig. $1 \mathrm{~b}$ and details). From the set of scores, we leverage routines from the bumphunter and limma packages to calculate a statistic and detect regions showing persistent change in ASM. We call these regions DAMEs (Fig. 1c). We estimate and control a regional FDR through permutations or by implementation of the Simes method [42].

\section{The ASM score \\ SNP-based ASM}

The most straightforward way of detecting SD-ASM from mapped reads is by assigning them to either of the alleles at each known heterozygous SNP. Methylation status is then determined for each allele-linked cytosine in the reads. We have used this strategy to calculate an SNP-based ASM score $\left(A S M_{\text {snp }}^{i}\right)$, and considered it to be the genuine form of SD-ASM, since it is derived from an extra layer of information, i.e., the genotype of an individual.

Ideally, the genotype should originate from genome sequencing; however, this type of data is scarce in large cohorts of samples. Therefore, we have employed BisSNP [33] to call SNPs directly from the BS-seq reads. The method uses Bayesian methods to infer strandspecific base calls, with SNP population frequencies as prior probabilities.

We extract the reads overlapping every heterozygous SNP in a VCF file with the GenomicAlignments R package [44], and for each read determine the methylation status of the CpG sites. Sites that are not in reads containing an SNP are not considered. We calculate $A S M_{s n p}^{i}$ for each CpG site $i$ contained in the reads of an SNP as

$$
\mathrm{ASM}_{\mathrm{snp}}^{i}=\left|\frac{X_{M}^{i r}}{X^{i r}}-\frac{X_{M}^{i a}}{X^{i a}}\right|
$$

where $X_{M}^{i r}$ and $X_{M}^{i a}$ correspond to the number of methylated reads from the reference $r$ allele, and the alternative $a$ allele. In practice, it makes no difference which allele is the reference or the alternative. $X^{i r}$ and $X^{i a}$ correspond to the total number of reads covering the reference and the alternative allele (see schematic diagram in Fig. 1b). The score ranges from 0 to 1 , where a score of 1 represents the scenario where one allele is completely methylated, and the other allele is fully unmethylated; a value of 0 means an equal proportion of methylated sites in both alleles. 


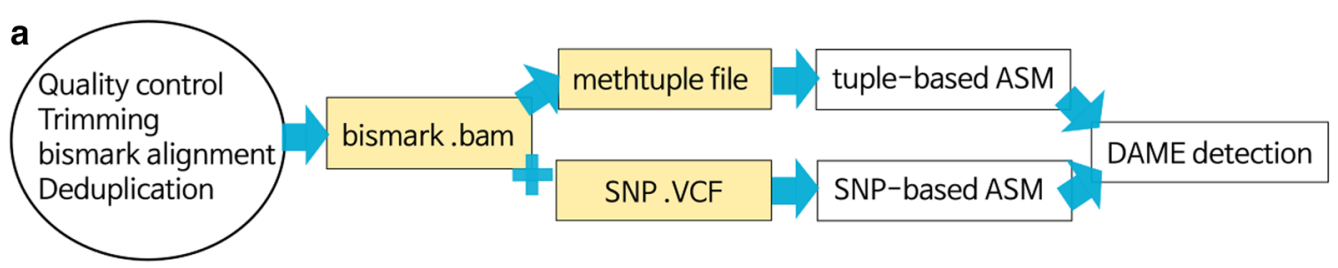

b
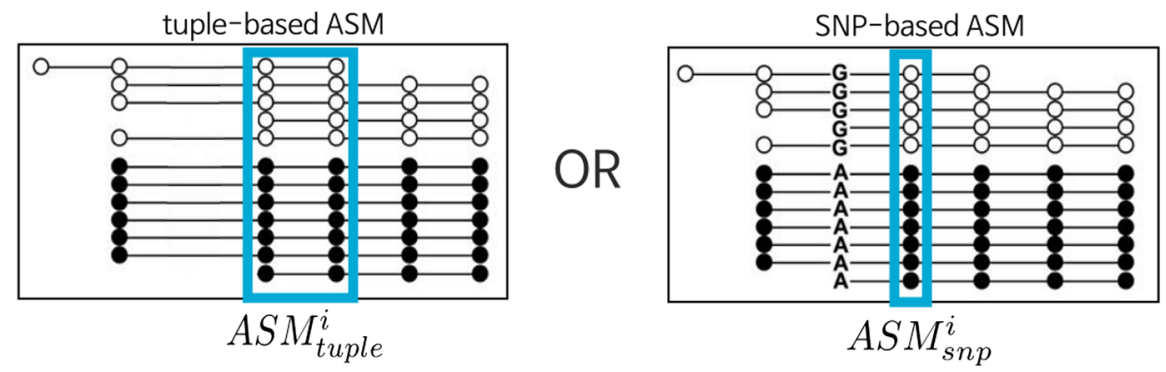

C
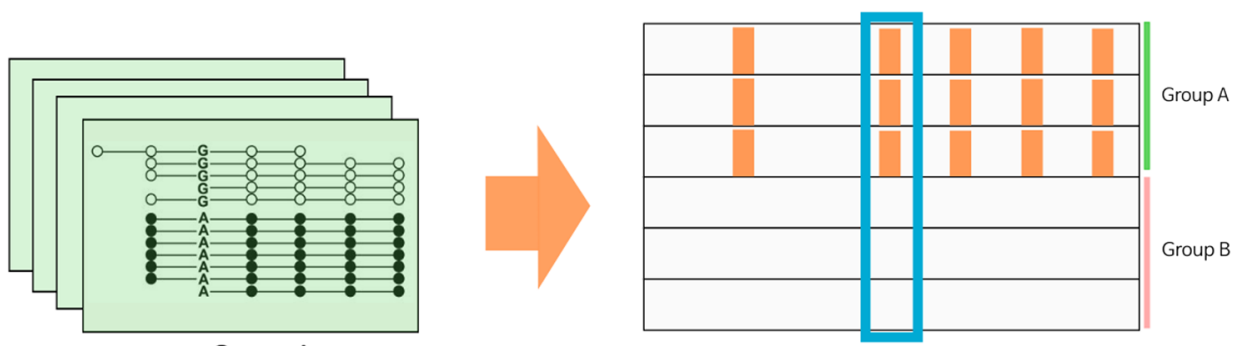

Group A

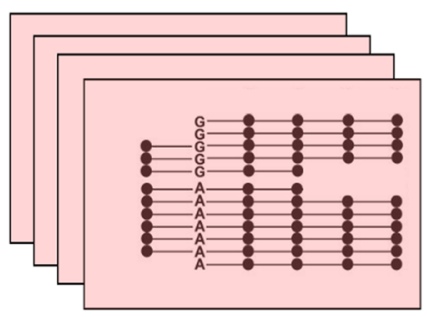

Group B
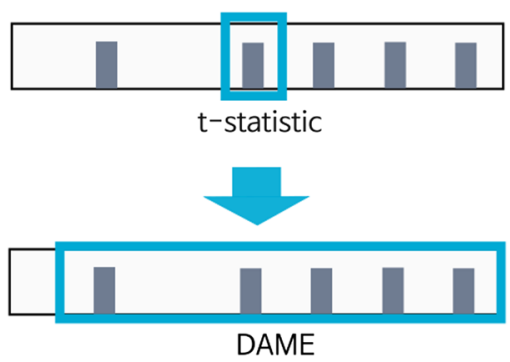

Fig. 1 The DAMEfinder pipeline. a Files necessary to run DAMEfinder are reported in yellow rectangles. White rectangles show the main $R$ outputs from DAMEfinder. Steps to be run before DAMEfinder are in the circle, i.e., fastq files undergo quality control and read alignment with bismark [43]. The resulting bam file is used to calculate an ASM score, which can be done in two ways: $\mathbf{b}$ (i) the tuple-based strategy that takes as input a beforehand created methtuple [41] file. The score is calculated based on the read counts of pairs of CpG sites. (ii) the SNP-based strategy, which takes as input both the bam file and a VCF file with heterozygous SNPs. Here, the score is calculated for each CpG site in the reads containing a SNP. c We determine differential ASM by calculating a statistic based on either the tuple ASM or the SNP-ASM (using limma [39]), which reflects the difference between two conditions (Group A vs. Group B) for each genomic position (tuple or site). DAMEs are defined based on this statistic, as regions of contiguous positions with a consistent change in ASM

\section{Tuple-based ASM}

Instead of restricting ASM detection to allele-linked reads, we can make use of an entire set of CpG sites to screen for different types of ASM. For this task, we designed a score under the assumption that pairs of $\mathrm{CpG}$ sites in the same DNA molecule (read) are correlated [45, 46], and that in a biallelic organism, intermediate levels of methylation could represent allele specificity, i.e., the proportion of methylated reads in a pair of CpG sites or tuple is close to 0.5 . We make this assumption knowing that other scenarios exist in which intermediate methylation can occur (e.g., cellular heterogeneity). Therefore, we consider this score only as a proxy for ASM.

We calculate the score as a weighted log-odds ratio: 


$$
\mathrm{ASM}_{\text {tuple }}^{i}=\left|\log _{10}\left\{\frac{\left(X_{\mathrm{MM}}^{i}+c\right)\left(X_{\mathrm{UU}}^{i}+c\right)}{\left(X_{\mathrm{MU}}^{i}+c\right)\left(X_{\mathrm{UM}}^{i}+c\right)}\right\} \cdot w_{i}\right|
$$

where $X^{i}$. corresponds to the number of reads covering a unique pair of $\mathrm{CpG}$ sites $i$, generated by running the methtuple tool. CpG sites in a pair can be methylated $\mathrm{MM}$, unmethylated UU, or mixed (UM or MU). A constant $c$ is added to every $X^{i}$ to avoid dividing by 0 . The $\log$-odds ratio is multiplied by a weight, $w_{i}$, which is set such that the ratio of MM:UU can depart somewhat from a 50:50 relation, while MM or UU tuples, which represent absence of allele specificity, are attenuated to 0 . This is calculated as

$$
w_{i}=P\left(0.5-\epsilon<\theta^{i}<0.5+\epsilon \mid X_{\mathrm{MM}}^{i}, X_{\mathrm{UU}}^{i}, \gamma\right)
$$

where $\epsilon$ represents the degree of allowed departure from a $50: 50$ ratio (i.e., 0.5 ), and $\theta^{i}$ :

$$
\theta^{i} \mid X_{\mathrm{MM}}^{i}, X_{\mathrm{UU}}^{i}, \gamma \sim \operatorname{Beta}\left(\gamma+X_{\mathrm{MM}}^{i}, \gamma+X_{\mathrm{UU}}^{i}\right),
$$

represents the moderated proportion of $\mathrm{MM}$ to $\mathrm{MM}$ + UU reads. It is based on a beta model, where $\gamma$ is a hyperparameter set to penalize fully methylated or fully unmethylated tuples, i.e., when the MM:UU balance goes farther from a 50:50 relation. In Fig. 2, we show 4 examples in which the score is calculated with and without $w_{i}$. According to our assumption, example 1 is the best case of ASM (Fig. 2a), yet we see for example, that examples 1 and 3 have the same absolute log-odds ratio (without $w_{i}$ ), but imposing the weight reduces the score in example 3 below that of example 1 (Fig. 2c). We also demonstrate how the $\mathrm{ASM}_{\text {tuple }}$ score is affected by the tuning of $\epsilon$ and $\gamma$ (Additional file 1: Figure S13A, B). $\epsilon$ has the most impact in the score, i.e., smaller departures from 50:50 result in lower values of $\mathrm{ASM}_{\text {tuple }}^{i}$, whereas only very large values of $\gamma$ (e.g. > 5) influence the distribution of $\mathrm{ASM}_{\text {tuple. }}^{i}$

\section{ASM score validation $S D-A S M$}

To test the $\mathrm{ASM}_{\text {tuple }}$ score, we used the $\mathrm{ASM}_{\text {snp }}$ score as an indicator of true SD-ASM, and calculated the $\mathrm{ASM}_{\text {tuple }}$ score, the allelicmeth and amrfinder scores, and a score representing absolute deviation from $50 \%$ methylation (methdeviation; see "Methods"), in a single normal tissue sample from the colorectal cancer (CRC) dataset (see "Methods"). We set CpG tuples with ASM $_{\text {snp }}$ above specific thresholds ( 0.5 and 0.8$)$ to be true SDASM. These resulted in 1284 and 69 CpG tuples with SDASM, respectively, which corresponded to $0.069 \%$ and $0.003 \%$ of all the tuples scanned in this sample.

Figure 3 shows the true-positive rate (TPR) and falsepositive rate (FPR) achieved by the 4 evaluated scores at different $\mathrm{CpG}$ tuples classified in 3 different coverage

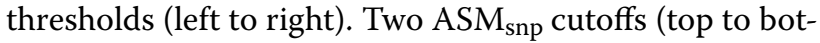
tom) were chosen as the truth. $\mathrm{ASM}_{\text {tuple }}$ was consistently more sensitive and specific than the other three scores, especially as coverage increased. Intermediate methylation values yielded comparable results; however, the $\mathrm{ASM}_{\text {tuple }}$ was able to detect more cases of "real" ASM in all combinations. allelicmeth increasingly failed as coverage and $\mathrm{ASM}_{\mathrm{snp}}$ value increased. amrfinder performed better than allelicmeth at higher true values. The distributions of the scores from each facet in Fig. 3 are in Additional file 1: Figures S11A, B and S12A-D.

\section{Chromosome X inactivation}

As an additional validation of the $\mathrm{ASM}_{\text {tuple }}$ score, we used the blood dataset (see "Methods") to compare healthy male and female samples. In principle, females should exhibit allele specificity in the X chromosome due to XCI and thus higher $\mathrm{ASM}_{\text {tuple values. Figure } 4 \text { shows }}$ the distribution of $\mathrm{ASM}_{\text {tuple }}$ values across all samples in the dataset, in chromosome 3 and chromosome X. From a whole genome perspective (Fig. 4a), there is little difference between males and females in $\mathrm{X}$ chromosome (mean of sample-means females: 0.13, males: 0.098), and practically no difference in chromosome $3(0.060,0.074)$. However, by focusing on $\mathrm{CpG}$ tuples located in promoter regions (1 kb upstream the transcription start site-TSS), we observed ASM values increased only in chromosome $\mathrm{X}$ of females (Fig. 4b; 0.30, 0.088).

\section{Imprinted ASM}

In the same blood dataset, we also compared the $\mathrm{ASM}_{\text {tuple }}$ scores from the promoters of imprinted genes reported in [11] (see "Methods") to the scores from the rest of the genome (Fig. 4c). As expected, ASM scores were higher in the tuples located within imprinted promoters, for both males and females.

\section{DAME detection}

As depicted in Fig. 1, after calculating $\mathrm{ASM}_{\text {tuple }}$ or $\mathrm{ASM}_{\mathrm{snp}}$ in the DAMEfinder pipeline, we continue to detect regions of persistent change in ASM between one condition to another within a cohort of samples. Change can occur as loss of ASM, when a reference group exhibits allele specificity across a region (high values of ASM), and the group of interest has this same region fully methylated, unmethylated, or with random methylation (low values of ASM). Change can also occur as gain of ASM, where the reference group does not have allele specificity and the group of interest does. We call regions such as this DAMEs (Differentially Allele-specifically MEthylated regions). 
a

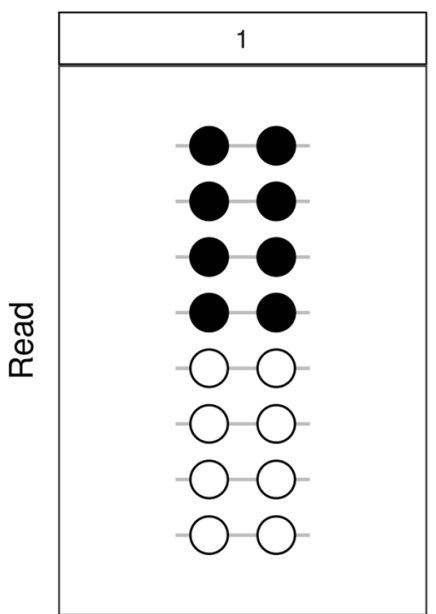

b

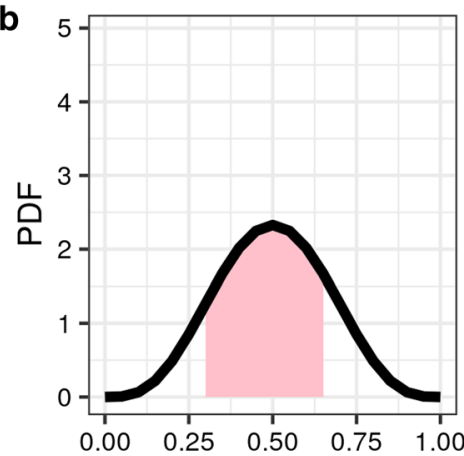

C
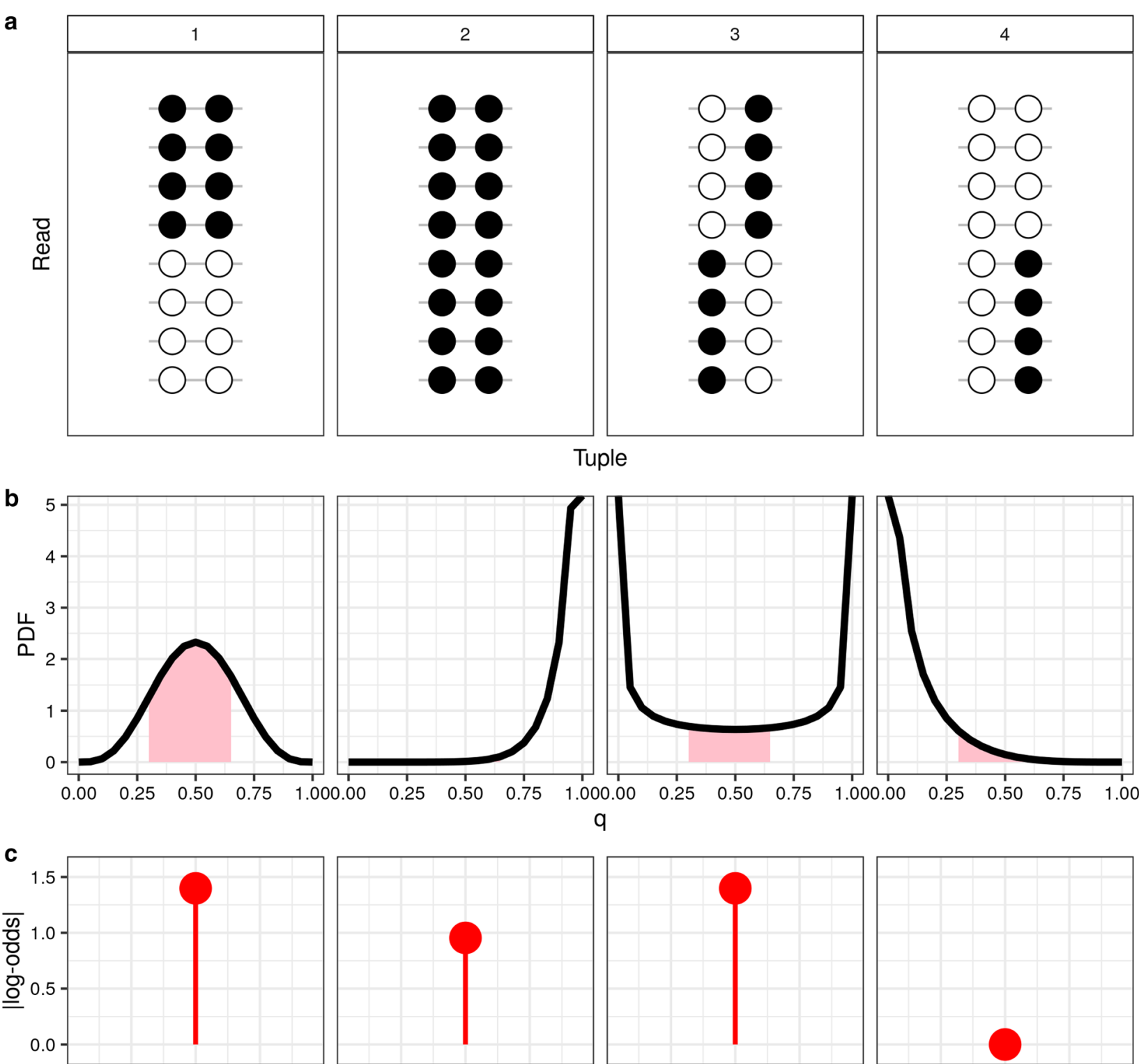

Tuple
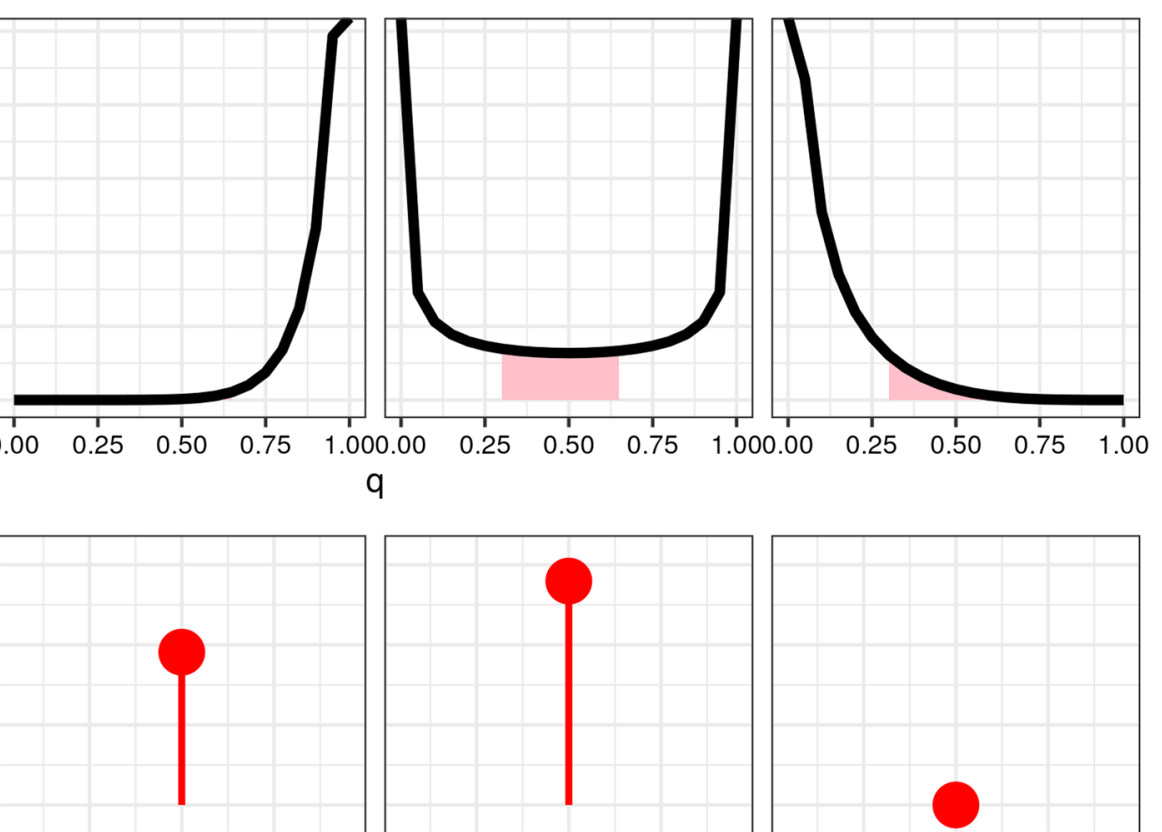

q
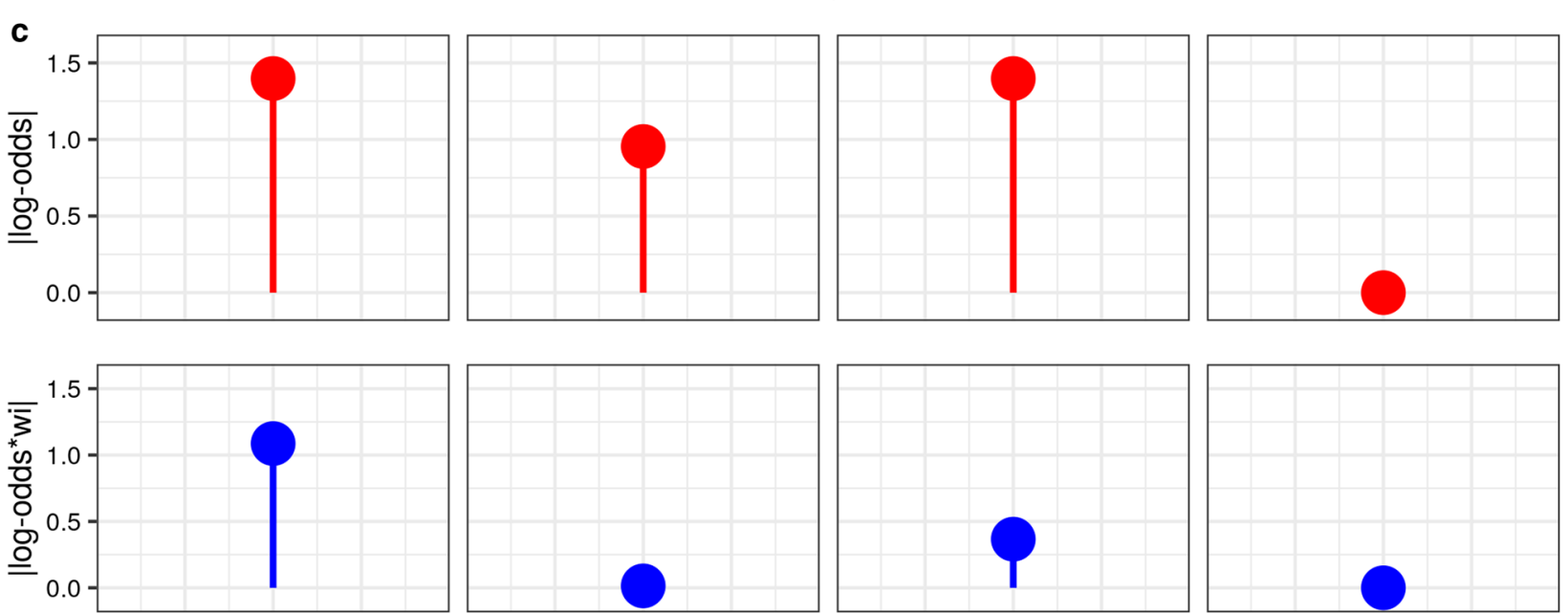

Tuple

Fig. $2 \mathrm{ASM}_{\text {tuple }}$ weighting strategy. We show 4 examples of read-patterns in a single tuple. a All cases have 8 reads covering a single tuple. Example 1 has $X_{M M}^{i}$ and $X_{U U}^{i}=4$, and $X_{U M}^{i}, X_{M U}^{i}=0$. Example $2 X_{M M}^{i}=8$, the rest of combinations 0 . Example $3 X_{U M}^{i} X_{M U}^{i}=4$, and example $4 X_{U U}^{i}$, $X_{U M}^{i}=4$. b Probability density function (PDF) for each Beta $\left(\gamma+X_{M M^{\prime}}^{i} \gamma+X_{U U}^{i}\right)$ of the 4 examples, when $\gamma=0.5$. Pink-shaded area corresponds to $0.5 \pm \epsilon$ when $\epsilon=0.2$. c Based on the PDF, we calculate the log-odds ratio (top), and the log-odds ratio multiplied by $w_{i}$ (bottom) 


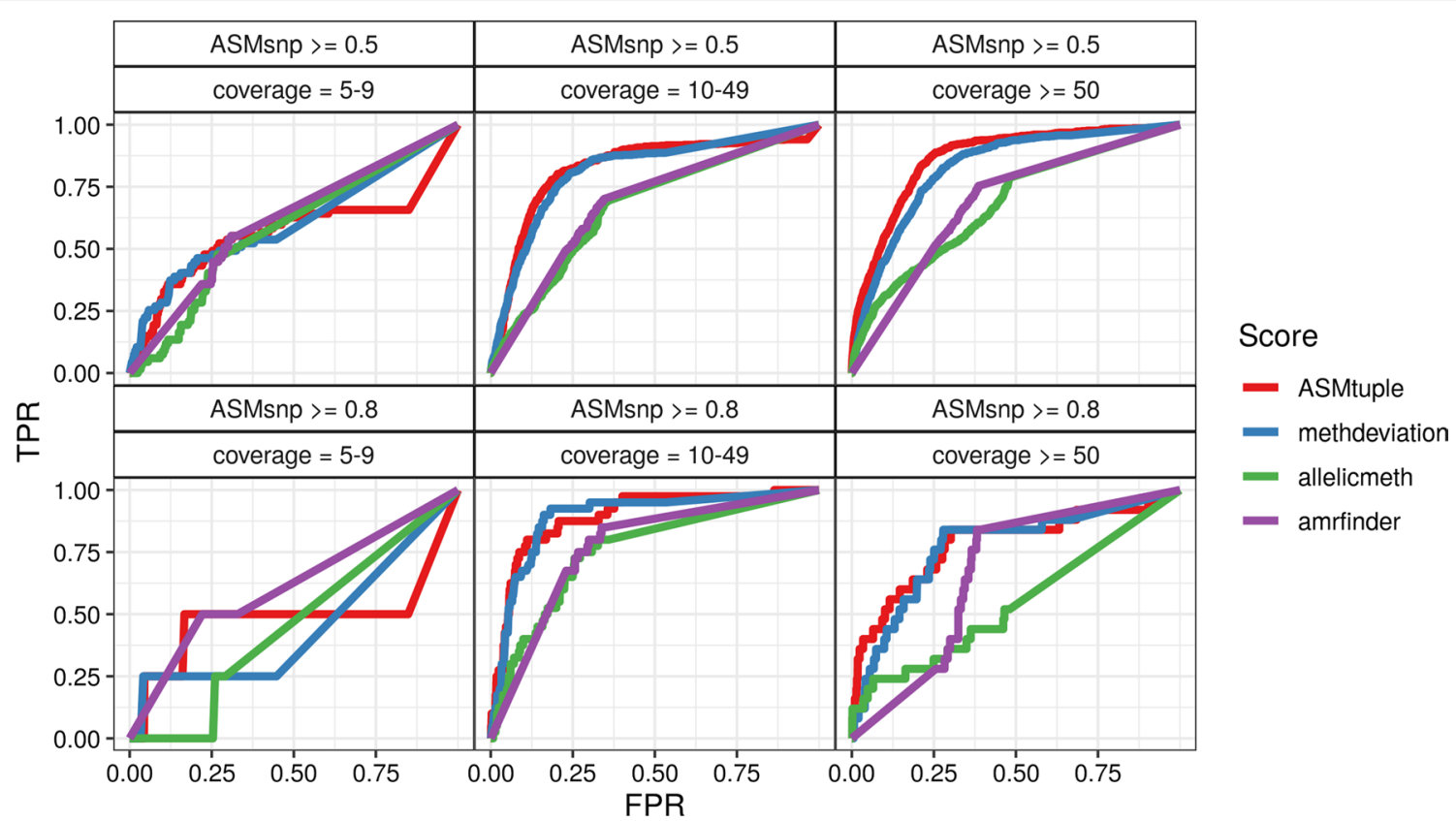

Fig. 3 Comparison of the ASM tuple Score to allelicmeth, amrfinder and methylation deviation, by considering ASM snp as true ASM. We calculated ASM tuple Scores (red), deviations from 50\% methylation (blue), allelicmeth scores (green), amrfinder scores (purple) in a sample of normal colorectal mucosa included in the CRC dataset. The scores were compared to each other by plotting the FPR against the TPR achieved. The plots are drawn for different intervals of read coverage (5-9, 10-49, $\geq 50)$, and different levels of the ASM $_{\text {snp }}$ score $(\geq 0.5, \geq 0.8)$, which is considered the "true" ASM. Overall AUCS (area under the curve) for the top three panels: $A_{S M}$ tuple $=0.83$, deviations from $50 \%=0.81$, allelicmeth $=0.66$, amrfinder $=$ 0.68. Overall $A \cup C s$ for the lower three panels: $A S M$ tuple $=0.82$, deviations from $50 \%=0.81$, allelicmeth $=0.64$, amrfinder $=0.72$

To detect DAMEs, we first obtain a regression coefficient $\beta_{i j}$ followed by a t-statistic using the $\mathrm{R}$ package limma [39] (see "Methods"), on the transformed $\mathrm{ASM}_{\text {tuple }}^{i}$ score, or on the $\mathrm{ASM}_{\mathrm{snp}}^{i}$ score, for each CpG position $i$ (tuple or site), across $j$ samples (see "Methods" for model).

We detect regions of contiguous CpG positions where $\beta_{i j}$ persistently deviates in the same direction from zero; this is done in two ways:

\section{Permuting bumphunted regions}

The regionFinder function from bumphunter is used to scan for regions $(R)$ where CpG sites close in proximity have $\beta_{i j}$ above a user-defined threshold $K$, which corresponds to a percentile of $\beta_{i j}$. For each region detected, the function also calculates an area $A=\sum_{i \in R}\left|\beta_{i j}\right|$. For the CRC dataset, we used the default value $K=0.7$, and distance between CpG positions up to $100 \mathrm{bp}$.

We assess significance of every region detected by assigning an empirical $p$ value. For every non-redundant permutation of the coefficient of interest (chosen from a column in the design matrix $X$ ), regionFinder is applied again. All the areas generated by all permutations are pooled to generate a null distribution of areas [47]. We define the $p$ values for each $R$ as the proportion of null areas greater than the observed $A ; p$ values are adjusted using the Benjamini-Hochberg method [48] from the stats R package [38].

\section{Cluster-wise correction}

Optionally, we define regions that exhibit changes in ASM by first generating clusters of CpG sites with clusterMaker. For each cluster, we aggregate all the CpG position $p$ values generated by limma using the Simes method [42], which is applicable when test statistics exhibit positive dependence [49]. As implemented in [50], we calculate

$$
p_{c}=\min \left\{n p_{(i)} /(i)\right\}
$$

where $p_{(1)}, \ldots, p_{(n)}$ are the ordered $p$ values of each CpG position $i$ in a cluster $c$, and $n$ is the number of CpG positions in the cluster. $p_{c}$ summarizes evidence against the null hypothesis that all $\mathrm{CpG}$ positions are not differential. We adjust $p_{c}$ as above.

\section{Evaluation of DAME detection}

We compared the different strategies to control FDR in the DAME detection pipeline, by applying them to a semi-simulated dataset and plotting the TPR and FDR 

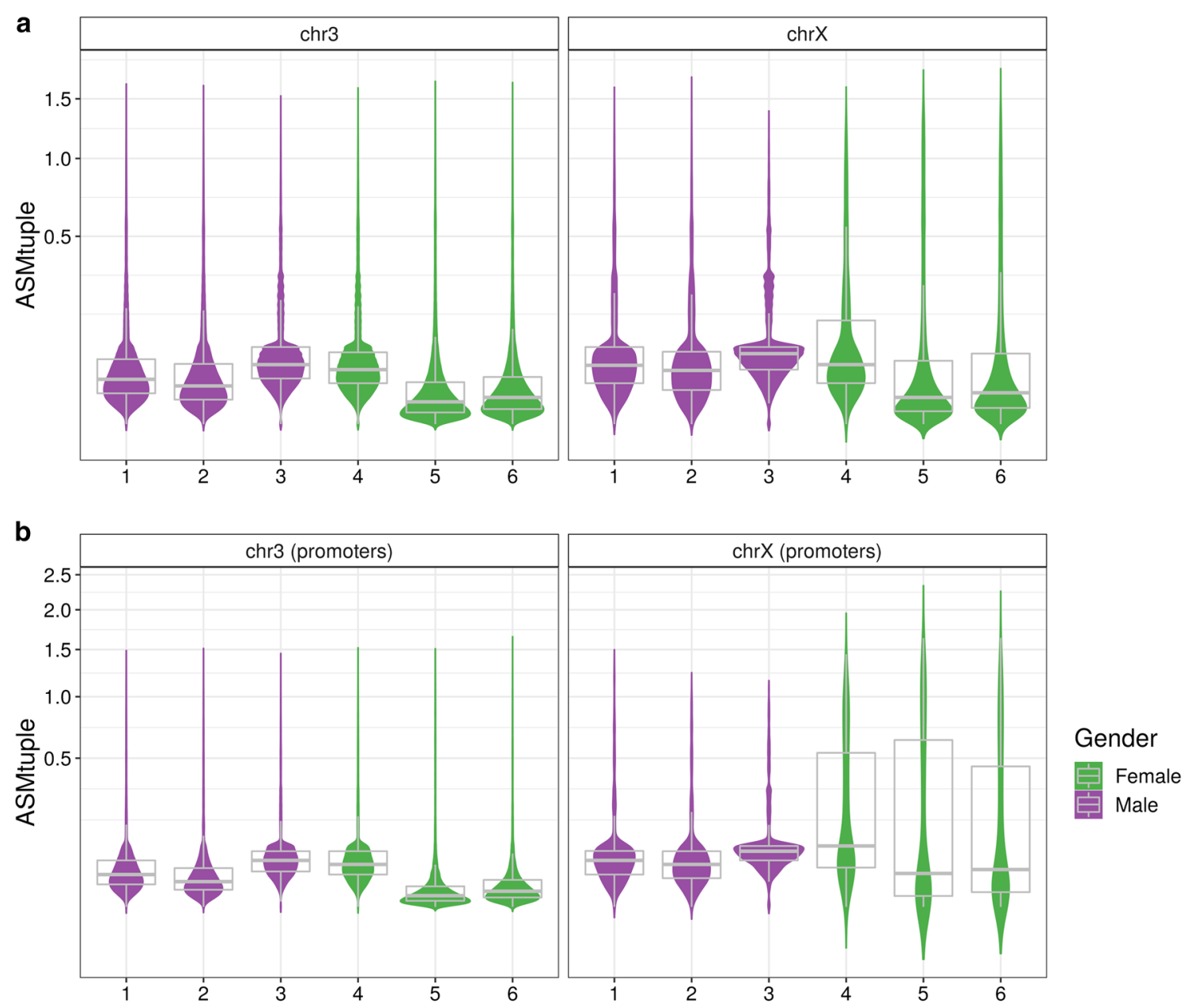

C

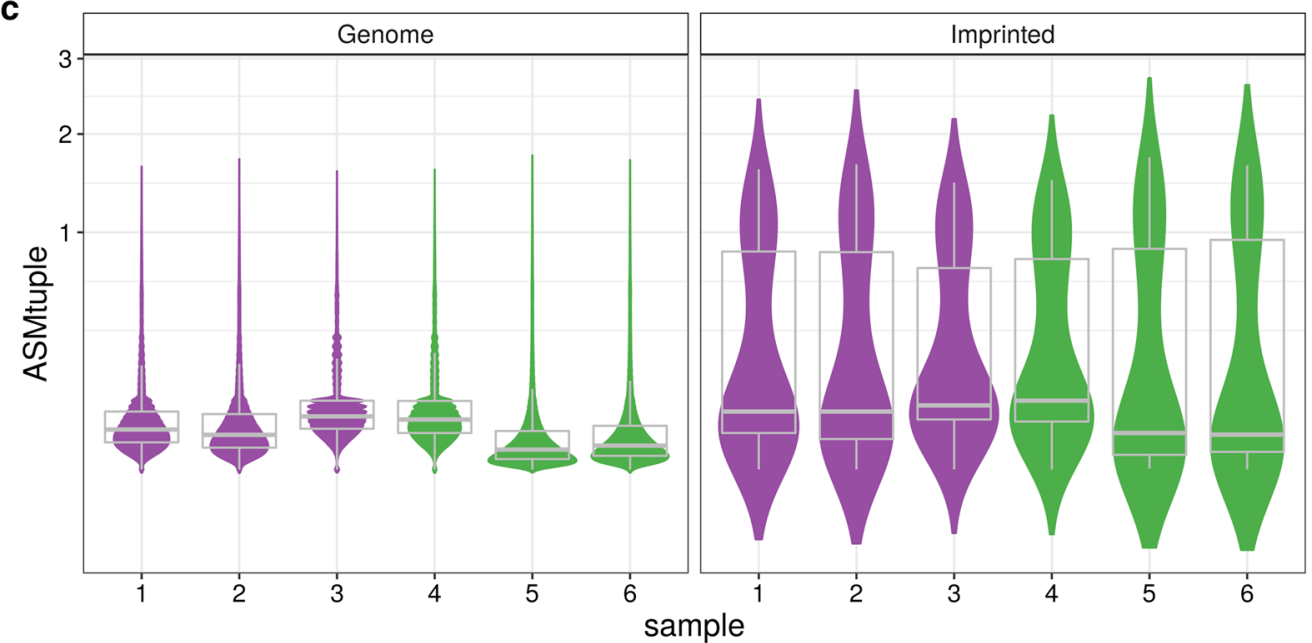

Fig. $4 \mathrm{ASM}_{\text {tuple }}$ distribution in the genome. We used XCl as a proof of concept for allele specificity in females. Data from the blood dataset

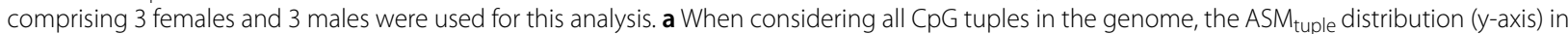
chromosome 3 and chromosome $X$ is similar in both genders. b When considering CpG tuples located in promoter regions (i.e., 1 kb upstream of

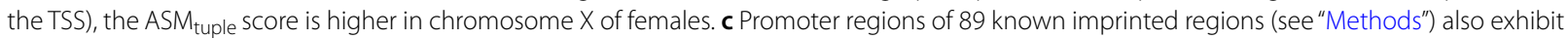
higher ASM $_{\text {tuple }}$ Compared to values in the rest of the genome. Y-axis in all plots is square-root transformed 


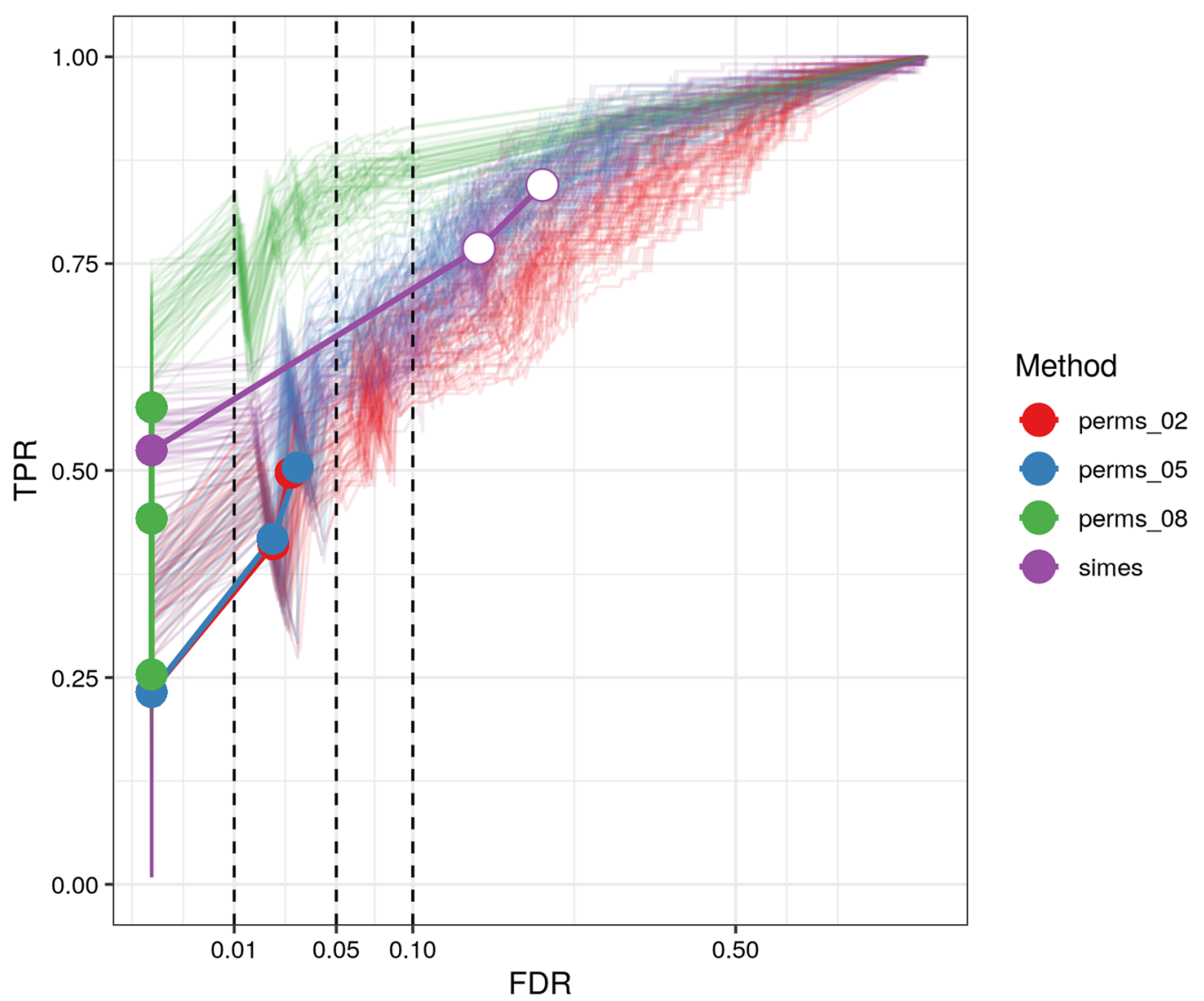

Fig. 5 FDR control of $p$ value assignment strategies. We plot the FDR against the TPR achieved by the two alternatives for assigning $p$ values to a DAME: the first by generating permutations and setting a threshold $K$ (see text) on the t-statistic (here $0.2,0.5,0.8$ ), the second using the Simes method. Lines are colored by strategy. Each strategy was run 50 times with the same simulation parameters. Colored circles indicate that the FDR achieved is smaller than the specified threshold (dashed lines at $0.01,0.05$ and 0.1 ), and white circles indicate the opposite. $x$-axis is square-root transformed

achieved at different adjusted $p$ value thresholds $(0.01$, $0.05,0.1$ ) (Fig. 5). We designed a small set of simulated DAMEs to evaluate the FDR control of the above strategies. We took 6 samples of normal tissue from the CRC dataset and calculated $\mathrm{ASM}_{\text {snp }}$ scores in each of them. We assumed these scores to be the $\mathrm{ASM}_{\mathrm{snp}}$ baseline in the simulation. Then, we divided the samples into two groups of three samples each, and for all the CpG sites covered by the 6 samples, we defined clusters of contiguous CpG sites. For each truly differential cluster, we added signal to a randomly determined subset of adjacent CpG sites (see "Methods" for more details).

Overall, the empirical $p$ value controlled the FDR, whereas the Simes method tended to be less conservative but more sensitive (Fig. 5 and Additional file 1: Figure S1 for same plot tested with different parameters).

\section{Discovery of DAMEs in colorectal cancer dataset}

We used a previously published dataset comprising 6 patients with diagnosed colorectal cancer, three with CIMP (CpG-Island Methylator Phenotype), and three without CIMP (see "Methods"); DNA from normal mucosa and cancer lesions was bisulfite-sequenced. We ran DAMEfinder on this dataset in both modes, therefore obtaining the $\mathrm{ASM}_{\mathrm{snp}}$ and $\mathrm{ASM}_{\text {tuple }}$ scores. After filtering for coverage (more than 5 reads) and for sites with more than $80 \%$ of samples covered, we obtained information for 43,420 CpG sites using the $\mathrm{ASM}_{\text {snp. Using }}$ the tuple score, we obtained summaries for 1,849,831 CpG pairs. Within the DAMEfinder pipeline, we generated multi-dimensional scaling (MDS) plots using each score (Fig. 6a, b), and observed that both scores are able to recover distinct CRC phenotypes. However using the $\mathrm{ASM}_{\text {tuple }}$ score, samples cluster according to tissue type (normals, CIMP cancer and non-CIMP cancer) (Fig. 6a), whereas using the $\mathrm{ASM}_{\text {snp }}$ score, only the two cancer 

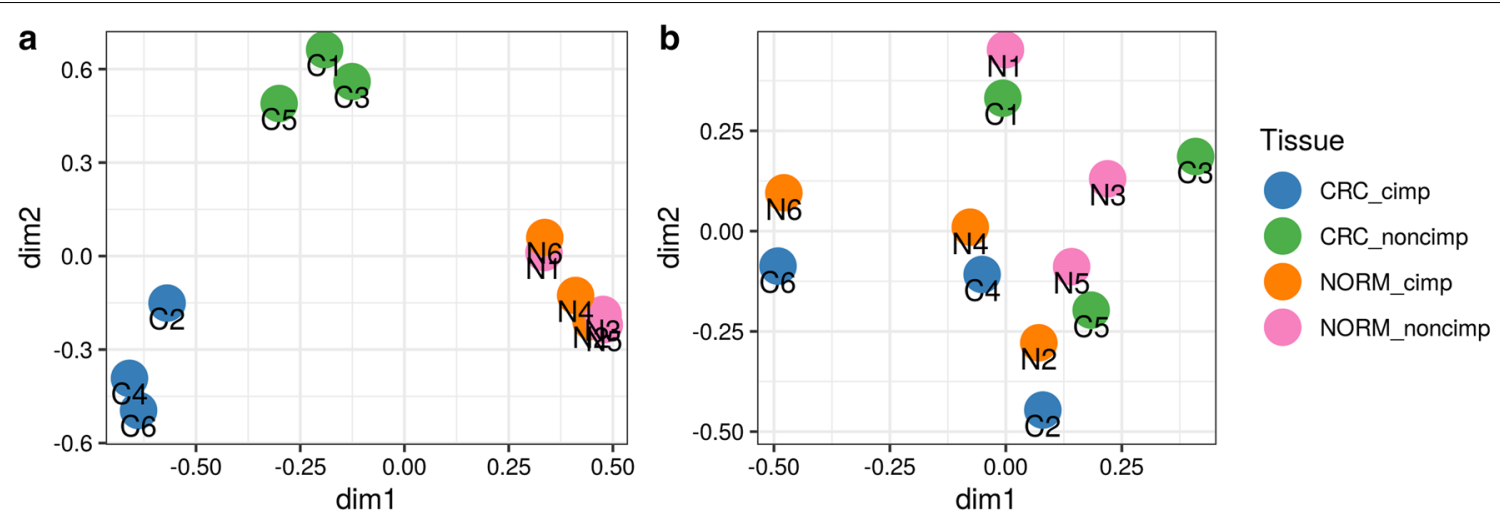

C

\section{Scores}
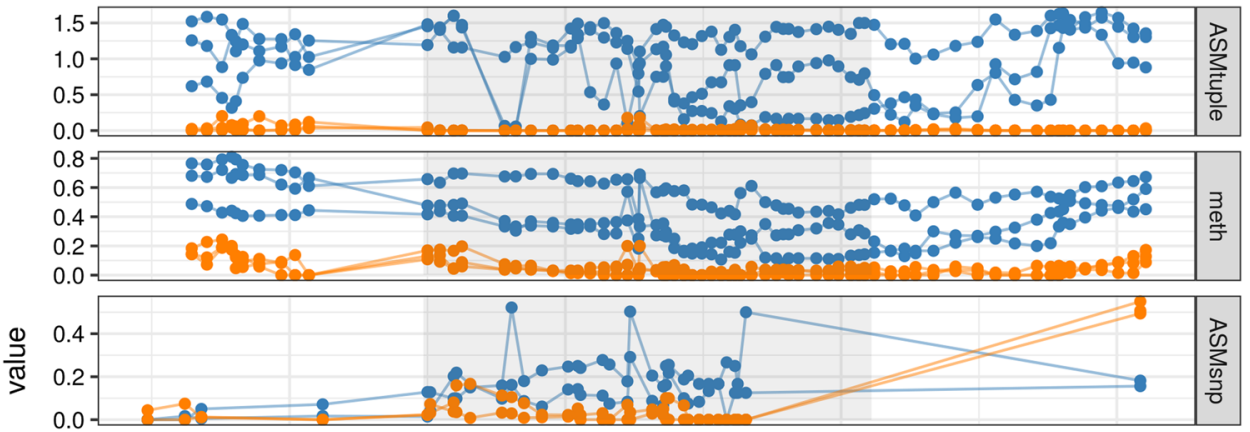

Tissue

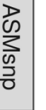

-- CRC_cimp

$\rightarrow$ NORM_cimp

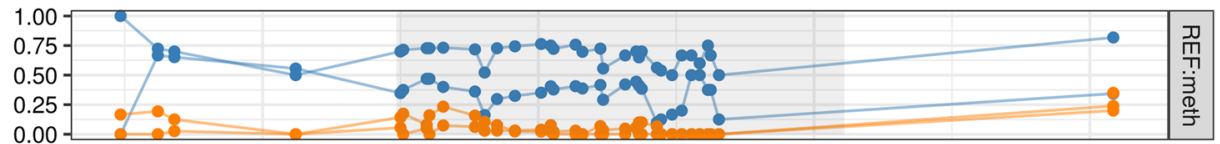

0.00

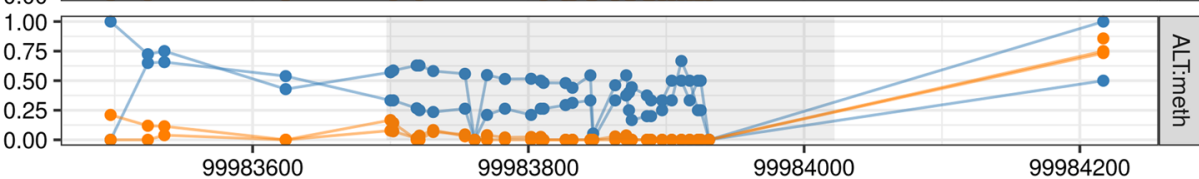

position

d

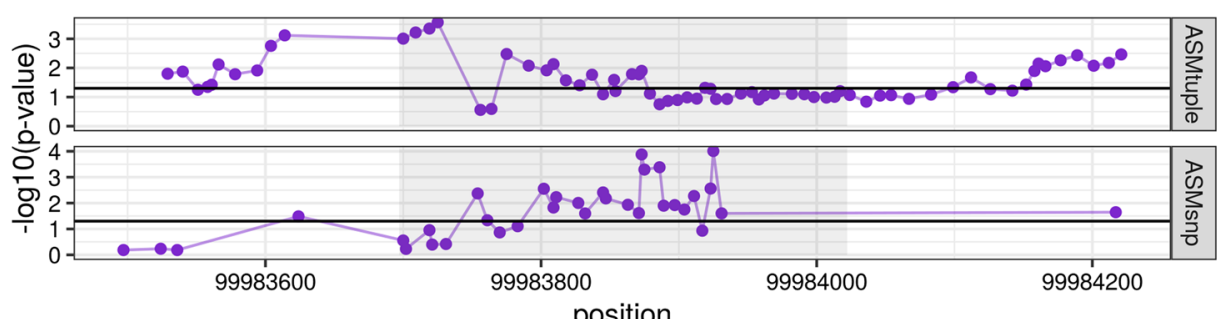

Fig. 6 ASM scores on the CRC dataset. a MDS plot of all the samples in the CRC dataset, based on all the the ASM tuple Scores. Scores were

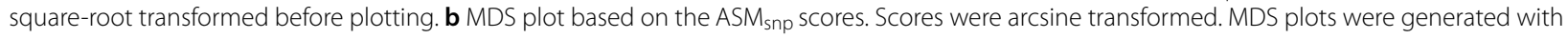
the plotMDS function from limma and the top 1000 most variable positions. N: normal mucosa; C: CRC. Each pair of samples from the 6 patients with CRC is numbered from 1 to 6. c A DAME detected in CIMP CRCs using the ASM tuple SCore shows a higher signal than using the ASM snp $_{\text {SCore. }}$ Region shown is located on chr9:99,983,697-99,984,022, shaded region in the center corresponds to the DAME. Tracks for methylation levels (meth) and methylation levels in reference and alternative alleles (based on SNP in chr9:99,983,812) are also shown. Points in ASM tuple and meth tracks correspond to intermediate positions between a pair of CpG sites. Points in the rest of tracks correspond to CpG sites. $\mathbf{d}-\log 10(p$ values) at each CpG position, calculated with ASM tuple and ASM snp. Black line is drawn at - log 10(0.05). Sample 4 (C4 and N4) does not appear in the last two tracks because it is not heterozygous at the evaluated SNP 
types are distinguishable, while the normal tissues cluster with their matched cancers (Fig. 6b).

We screened for DAMEs comparing cancer to normal tissue, in CIMP and non-CIMP independently. Both SNP and tuple-based scores were calculated, and for each separately, DAMEs were detected using the Cluster-wise correction (Additional file 1: Figure S2 for $p$ values of both Cluster-wise correction and Permutations). When using the $\mathrm{ASM}_{\text {snp }}$ score, we could not detect DAMEs with an adjusted $p$ value below 0.05. Using the $\mathrm{ASM}_{\text {tuple }}$ score, we were able to detect 4051 DAMEs in the CIMP samples (versus matched normal samples), and 258 in the non-CIMP samples. We noticed that regions detected using $\mathrm{ASM}_{\text {tuple }}$ were also detected using $\mathrm{ASM}_{\mathrm{snp}}$, but with lower strength of signal and with $p$ values above a cutoff of 0.05 (one example in Fig. 6c), and other regions showing contradicting changes in ASM (one example in Additional file 1: Figures S3, S4). Although the latter was rare (from all the 2219 DAMES detected in CIMP using $\mathrm{ASM}_{\text {snp }}$, without a $p$ value cutoff, only $0.36 \%$ disagreed on the ASM change), the cases we did find, overlapped tuple-DAMEs with the highest $p$ values, e.g., regions at the bottom of the DAME list. In addition, we found DAMEs corresponding to known regions exhibiting loss of imprinting in cancer, including those in the genes MEG3, H19, and GNAS [13, 51] (Fig. 7).

Considering the high number of DAMEs detected in the CIMP contrast compared to the non-CIMP contrast, we thought this could be a consequence of hypermethylation in CIMP [52], and so a typical DMR (differentially methylated region) analysis would be able to detect these same regions. To corroborate this, we performed a DMR analysis on the CIMP and non-CIMP contrasts using the dmrseq R package [47] (Additional file 1: Figure S5 for top DAMEs and DMRs per comparison). We found that from the 6753 DMRs (5,040 hypermethylated, 1713 hypomethylated) detected in the CIMP comparison, 2285 overlap with DAMEs (hypermethylated DMRs = $32 \%$, hypomethylated DMRs $=1.7 \%$ from total DMRs), and from 13,220 DMRs in the non-CIMP comparison, only 164 overlap (hypermethylated DMRs $=0.57 \%$, hypomethylated DMRs $=0.66 \%$ ) (Table 1$)$.

Because of this overlap, we conclude that a proportion (1146 [28\%] in CIMP, 93 [36\%] in non-CIMP) of DAMEs would not be detected via a typical DMR analysis. Figure 8 shows 4 examples of DAMEs missed by the DMR detection. In principle, these regions exhibit differential methylation according to the global methylation levels (bottom panels of each region); however, the hypermethylation reaches intermediate values, which might not represent a sufficiently high effect size to be detected. In the context of differential ASM, these intermediate values are highly scored, based also on the allele specificity of the change. Therefore, even though these are not highly ranked DAMEs, they were still detected as such.

\section{Discussion}

We have developed a scoring method that provides a measure of allele-specific methylation, and developed a method (DAMEfinder) that detects regions that display loss or gain of allele-specific methylation, by leveraging existing methods into a single framework. We offer the possibility to detect regions exhibiting ASM based on genotype information $\left(\mathrm{ASM}_{\mathrm{snp}}\right)$, or independent from it $\left(\mathrm{ASM}_{\text {tuple}}\right)$. The latter offers a novel approach for identifying different types of ASM, such as imprinted, nonimprinted, XCI, and new types yet to be described.

We have considered the $\mathrm{ASM}_{\text {snp }}$ score as genuine SDASM, and calculated it using heterozygous SNPs. We employed Bis-SNP to extract the SNPs from the BS-seq reads. The methods' accuracy (as occurs with normal SNP callers) requires a high read depth, because information from both strands of DNA is necessary to infer if a cytosine has been bisulfite-converted or not. The authors of the tool found that heterozygous SNPs require a minimum of $10 \mathrm{X}$ coverage to be accurately called $(80 \%$ sensitivity), and as depth is gradually increased to $30 \mathrm{X}$, sensitivity reaches $100 \%$. For our colorectal cancer analysis we only used coverages above 10X, and observed that increasing coverages did not affect our $\mathrm{ASM}_{\text {snp }}$ score (Additional file 1: Figure S7D). We also observed that the ROC curves from Fig. 3 did not change in the last two coverage groups ( $10-49$ and $\geq 50$ ).

Regarding the performance of the SNP-independent scores (allelicmeth, deviations from 50\% methylation and $\left.\mathrm{ASM}_{\text {tuple }}\right)$, we observed that $\mathrm{ASM}_{\text {tuple }}$ showed favorable performance at identifying individual cases of SD-ASM at sites with different coverage levels. The scaled methylation also demonstrated high sensitivity and specificity, and as the true SD-ASM score ( ASM $\left._{\text {snp }}\right)$ and coverage increased, results were close to those of the $\mathrm{ASM}_{\text {tuple }}$ score. Nonetheless, the advantage of using the $\mathrm{ASM}_{\text {tuple }}$ score is the flexibility in its implementation; in specific, the weight that is added to the log-odd ratio can be easily adapted as described in "Methods".

In contrast, the allelicmeth score reduced its performance when the true ASM value was increased. As for amrfinder, we believe defining ASM as regional is a nice implementation in this method, and can make ASM interpretation and visualization easier. However, the definition of regions is done for each sample independently, and this does not allow for a direct comparison between samples. This is the main reason why our ASM scores are not regional. Our method focuses on obtaining regions of consistent change in ASM between conditions relative to 


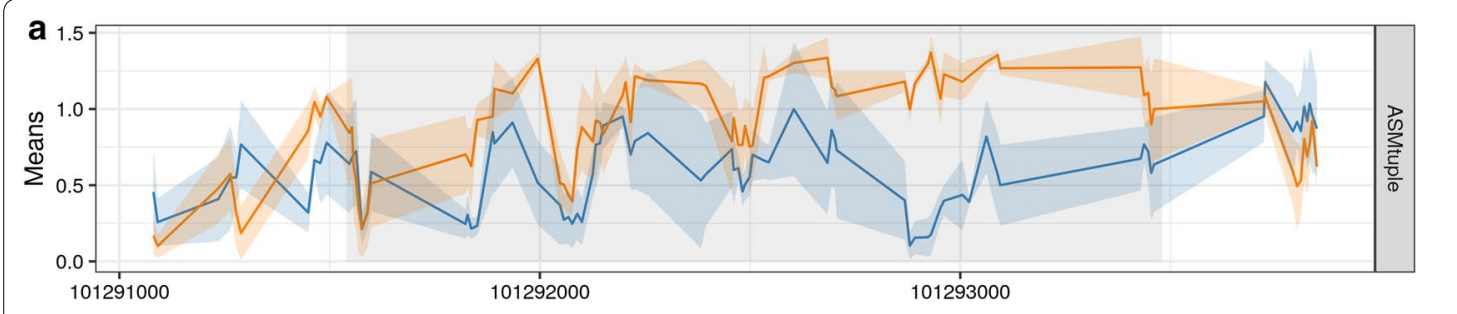

\section{Tissue}

- CRC_cimp

- NORM_cimp

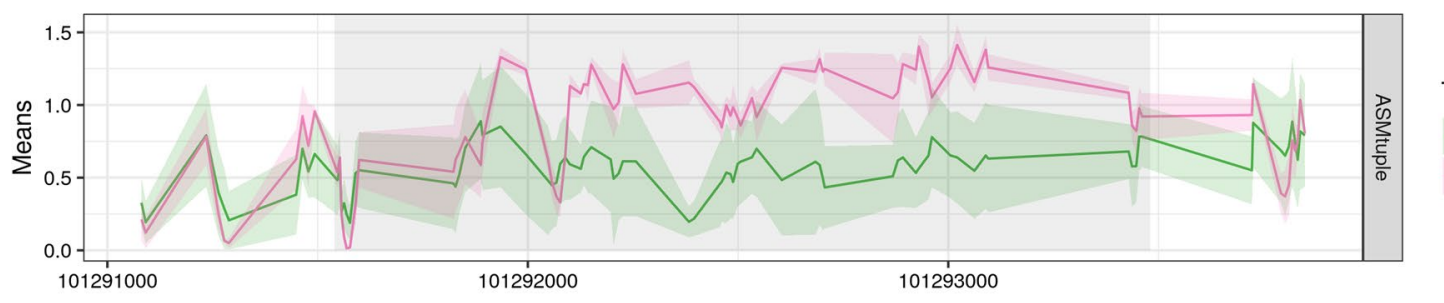

Tissue

- CRC_noncimp

_ NORM_noncimp

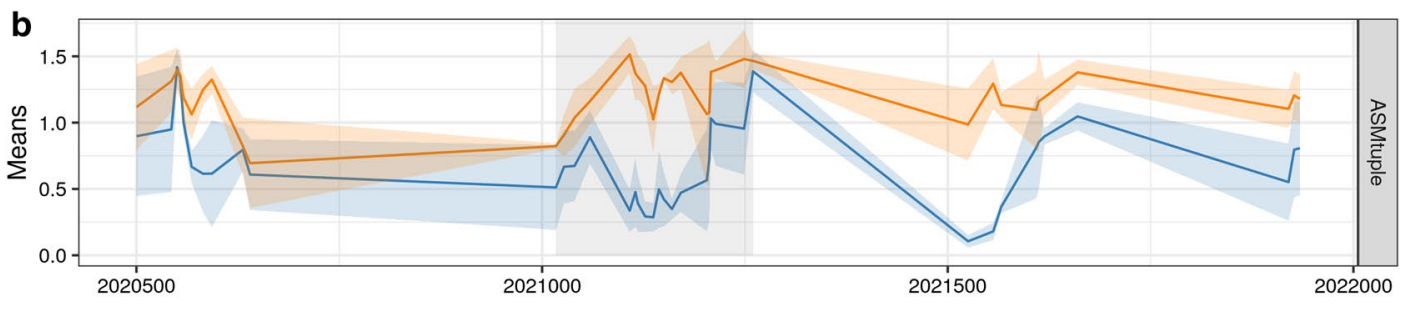

Tissue

- CRC_cimp

- NORM_cimp

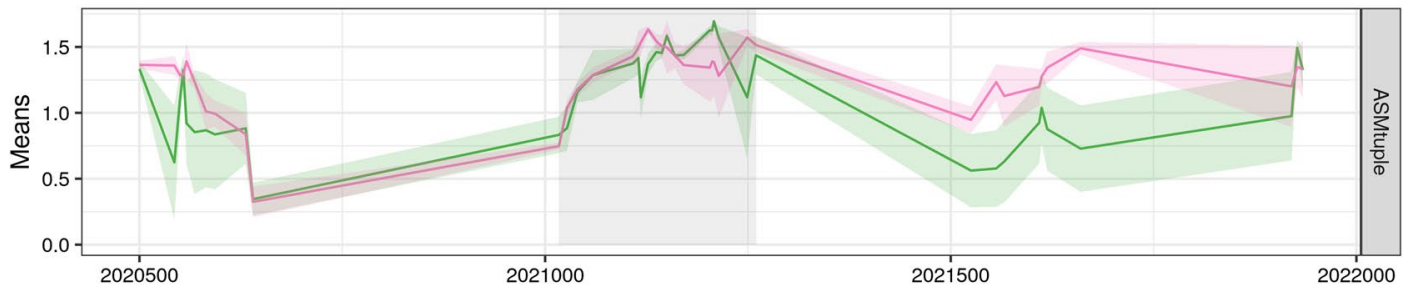

Tissue

- CRC_noncimp

- NORM_noncimp

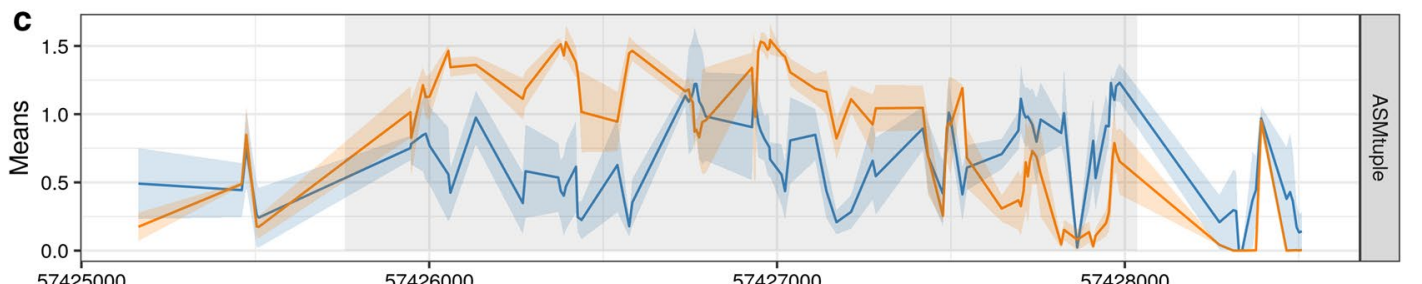

Tissue

- CRC_cimp

- NORM_cimp

57425000

57426000

57427000

57428000

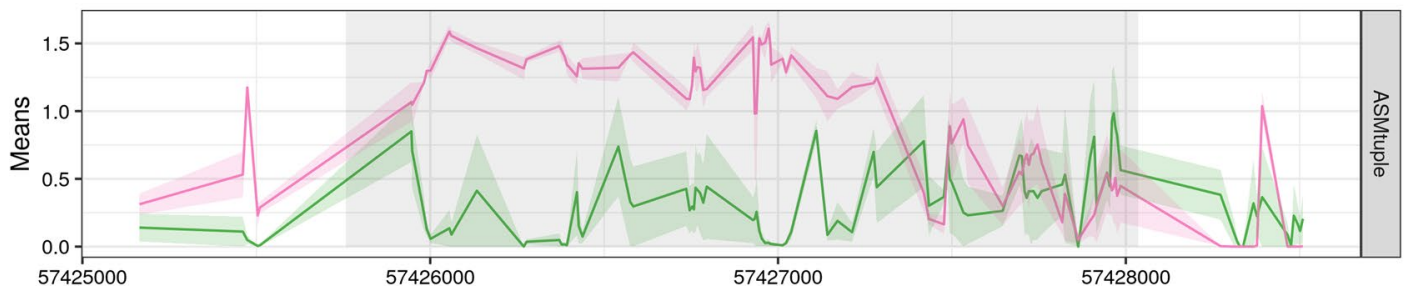

Tissue

- CRC_noncimp

- NORM_noncimp

Fig. 7 DAMEs overlapping known loci exhibiting loss of imprinting in colorectal cancer. a DAME located in chr14:101,291,540-101,293,480, upstream the imprinted MEG3 gene. The loss of imprinting was significant in both types of CRCs. b DAME located in chr11:2,021,017-2,021,260, upstream the imprinted H19 gene. Loss of imprinting only occurred in CIMP CRCS. c DAME in the GNAS gene located in chr20:57,425,758$57,428,036$. Loss of imprinting was detected in both types of CRCs. Y-axis in all panels corresponds to ASM tuple means. Lines connect means at intermediate positions between a pair of CpG sites. Shared areas correspond to confidence intervals at each position (standard errors of the mean) 
Table 1 DMRs overlapping DAMEs

\begin{tabular}{llcccc}
\hline & DMR state & Total DMRs & DMRs with DAMEs & DAMEs with DMRs & Gain/loss ASM \\
\hline CIMP & Hyper & 5040 & 2171 & 2789 & $2694 / 95$ \\
\multirow{2}{*}{ Non-CIMP } & Hypo & 1713 & 114 & 116 & $88 / 28$ \\
& Hyper & 3187 & 76 & 77 & $61 / 16$ \\
& Hypo & 10,033 & 88 & 88 & $64 / 24$ \\
\hline
\end{tabular}

Hyper- or hypo-methylated DMR refers to the increase or decrease of methylation in cancers in comparison with paired normal samples, while gain or loss of ASM refers to whether cancers have more or less allele specificity than paired normal samples

the variability, which in turn implies consistent ASM in the majority of samples from an experimental condition.

Our ASM tuple score was able to distinguish female from male samples based on XCI, which we have considered as another case of true ASM. When analyzing the entire genome, we did not find differences between males and females. The fact that the entire female chromosome $\mathrm{X}$ does not contain high ASM, or that the global distribution of methylation is not skewed toward intermediate values has been shown before [53]. In addition, the presence of genes escaping XCI may also affect global ASM. It is known that $15 \%$ of genes escape XCI, and an additional $10 \%$ varies in the inactivation state among the female population [54]. Therefore, a mixture of ASM scores in females is an accurate reflection of the complex dynamics of XCI.

We were also able to validate the score by comparing the promoters of 89 known imprinted genes with the rest of the genome. We observed an increase in the ASM of imprinted genes, with a bimodal distribution of ASM scores. This can be a reflection of tissue or cell type specificity in imprinted genes, meaning not all known imprinted genes show ASM throughout the somatic cell lineage, as is traditionally assumed [55]. Studies have reported tissue and cell type-specific allelic expression $[56,57]$ and tissue-specific ASM [23] in known imprinted genes, supporting our finding that imprinting is not equally maintained in all genes in every tissue and/or cell type.

Although the $\mathrm{ASM}_{\text {tuple }}$ score is able to recover different cases of ASM, we acknowledge that there are other cases not linked to ASM, such as cellular heterogeneity, in which a proportion of reads are fully methylated, and the rest are fully unmethylated. With the technology we have used (BS-seq), it is very difficult to discern heterogeneity from real ASM in some locations, and we believe that to identify ASM, single-cell BS-seq (scBS-seq) data may become the most suitable high-throughput technology. Previous studies have shown the use of scBS-seq to detect heterogeneity within a single cell type [58] and cell states [59]. However, the accurate detection of methylation from scBS-seq is still a difficult task, mainly due to the extensive DNA damage from the bisulfite treatment.
There are currently around 21 different protocols to profile single-cell DNA methylation, mostly bisulfite-based, each one aiming at improving recovery of CpGs and mapping efficiency [60]. However, it has not been established how these methods compare to each other, and a consistent framework for their data analysis does not exist, as is the case for bulk BS-seq protocols. Therefore, there is still work ahead to precisely quantify ASM using scBS-seq.

Another limitation arises when considering cancerous tissue samples, because of high intra-tumor heterogeneity of several biological features, including cellular morphology and gene expression [61]. Our method does not account for this additional variability, and we recognize this as a limitation. However, we believe the ASM scores are still robust enough to detect allelic patterns as shown by the recovery of the colorectal cancer subtypes in Fig. 6 and that even changes in cell composition, which would also affect DMR detection, can be interesting events to understand.

Regarding DAME detection, we offer two strategies that differ in the statistical stringency. In our experience, fewer regions are obtained by permuting the group labels, since the FDR control is more conservative. However, more regions can always be detected by setting the $K$ threshold lower, while still controlling the FDR. The Cluster-wise correction, or Simes method, is less conservative, and therefore can be used as an alternative to extract more detection power. This is likely because of the global hypothesis tested at each DAME, where at least one $\mathrm{CpG}$ site in a region is changed.

We applied DAMEfinder to a real dataset to detect DAMEs in CIMP and non-CIMP cancers (versus paired normal samples). We found that the $\mathrm{ASM}_{\text {tuple }}$ and $\mathrm{ASM}_{\text {snp }}$ scores are consistent in describing the CIMP status of samples, but as expected, the $\mathrm{ASM}_{\text {snp }}$ score was dominated by SD-ASM, because its calculation relies on the heterozygous SNPs of each sample; paired samples thus clustered with each other not by tissue, as observed with the $\mathrm{ASM}_{\text {tuple }}$ score. In addition, $\mathrm{ASM}_{\text {tuple }}$ typically detected more DAMEs, which we attribute to two reasons. First, there are $\sim 40 \times$ more places in the genome where $\mathrm{ASM}_{\text {tuple }}$ can be calculated. Second, because the 


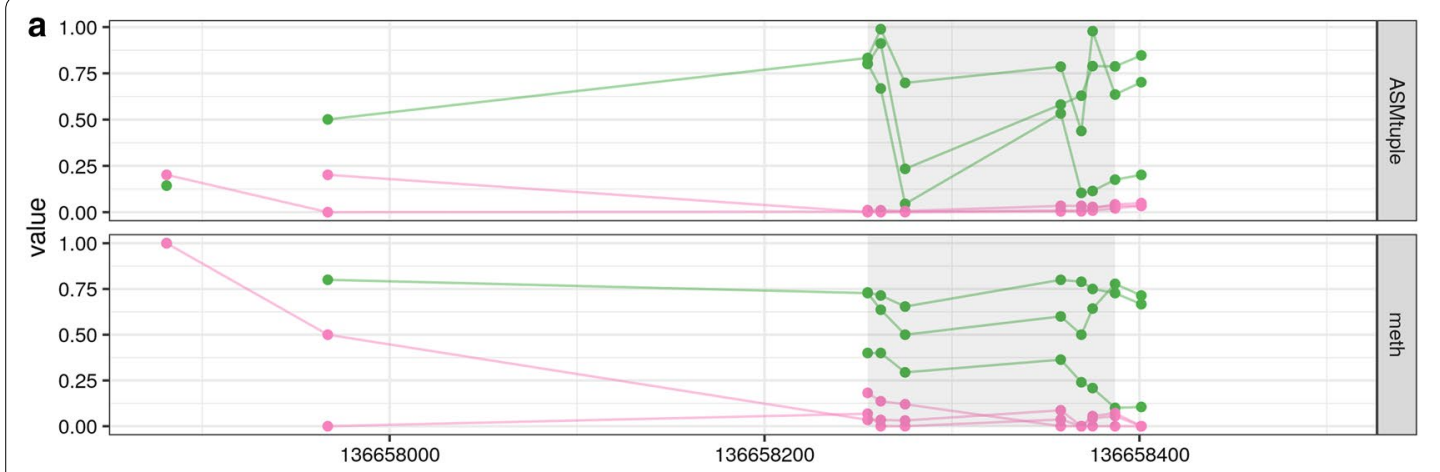

Tissue

- CRC_noncimp

- NORM_noncimp

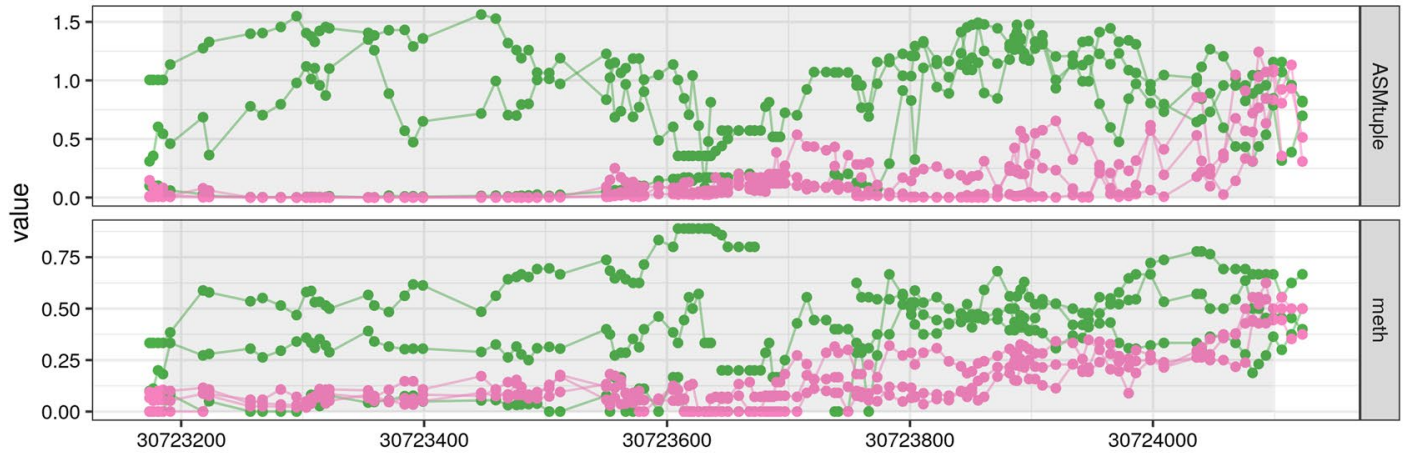

Tissue

- CRC_noncimp

- NORM_noncimp

b

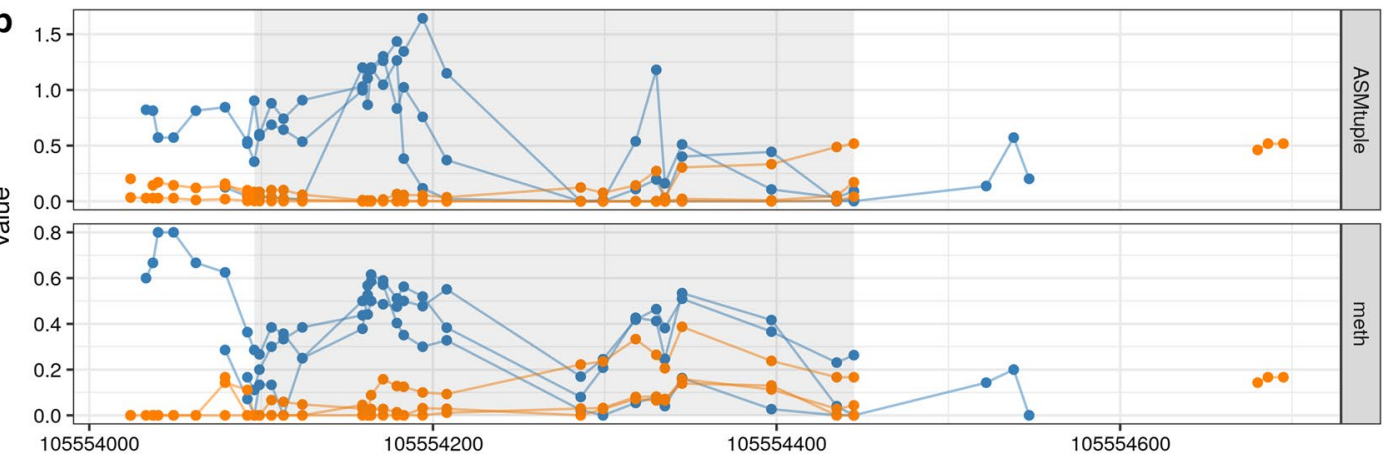

Tissue

-- CRC_cimp

- NORM_cimp

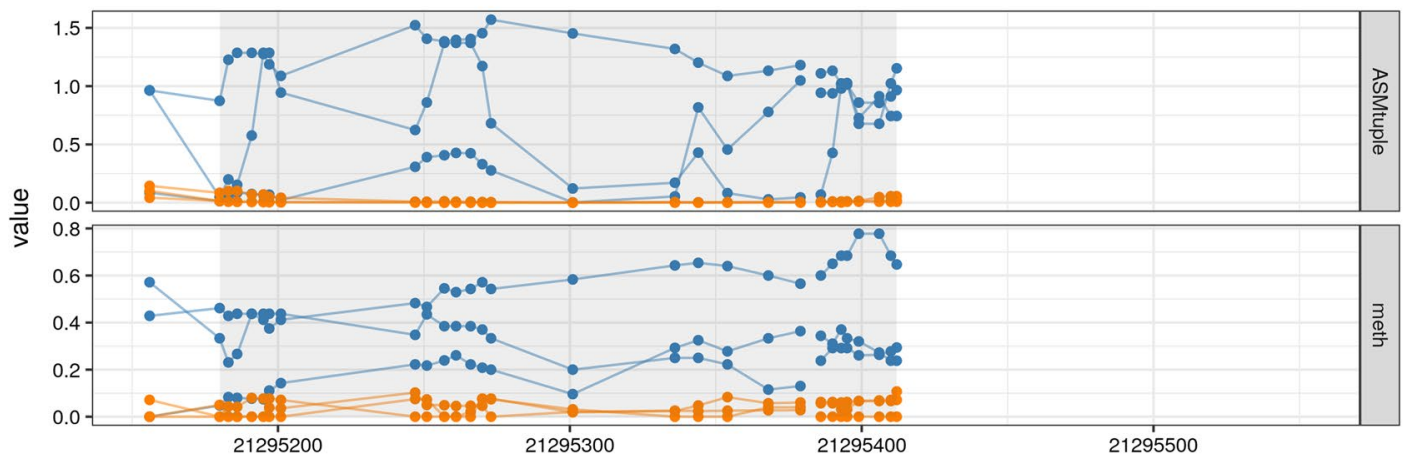

Tissue

- CRC cimp

- NORM cimp

Fig. 8 DAMEs not detected as DMRs. a Two different DAMEs in non-CIMP, the first located in chr9:136,658,255-136,658,387, and the second located in chr4:30,723,185-30,724,099. b Two different DAMEs in CIMP, the first in chr14:105,554,096-105,554,445; the second in chr16:21,295,18021,295,412. Y-axis corresponds to ASM tuple or methylation. Points correspond to intermediate positions between a pair of CpG sites 
tuple score is a more general calculation, i.e., it quantifies the mixing of methylated and unmethylated reads, instead of relying on allele information.

We also compared the DAME detection to a typical DMR analysis of the same samples, and found that DMRs detected may or may not include DAMEs. Most DMRs overlapping DAMEs were hypermethylated in CIMP cancers, which led us to conclude that most DAMEs reflected gain of ASM from a low methylation baseline. This result shows how differential ASM is a more refined definition of differential methylation, and can therefore provide additional information regarding methylation disruptions in disease (or different conditions).

\section{Conclusion}

Cytosine methylation restricted to only one allele, i.e., ASM, is a particular pattern of methylation that should be approached differently than the rest of the human methylome. We have designed DAMEfinder to screen for ASM and identify regions of differential ASM. The latter can be viewed as a special case of differential methylation. Previous studies have quantified ASM within one sample; however, to our knowledge, there is no method that identifies loss or gain of ASM between conditions. DAMEfinder fills this gap. Studying changes in ASM can help us understand epigenetic processes in development and diseases. To this aim, further studies are necessary to associate ASM to allele-specific gene expression and to verify whether gain or loss of ASM would affect gene dosage and eventually phenotypes.

\section{Methods}

The code used to generate the article figures and data processing is available from https://github.com/markr obinsonuzh/allele_specificity_paper. The $\mathrm{R}$ package is available from https:/github.com/markrobinsonuzh/ DAMEfinder.

\section{Datasets}

\section{Colorectal cancer (CRC) dataset}

The CRC dataset came from our published study [52] describing the progression of a methylation signature from pre-cancerous lesions to colorectal cancer tissue in two types of CRC. We used 12 samples from 6 patients with sporadic cancer (arrayexpress accession number: E-MTAB-6949, Table 2). For each sample, DNA from both CRC lesion and normal mucosa was bisulfitetreated and sequenced according the Roche SeqCapEpi CpGiant protocol, where only DNA captured by probes was sequenced. We analyzed 12 files in total. For details on data generation refer to [52].
Table 2 Colorectal cancer sample characteristics

\begin{tabular}{llllll}
\hline Sample ID & $\begin{array}{l}\text { CIMP } \\
\text { status }\end{array}$ & Sex & $\begin{array}{l}\text { Number } \\
\text { of mapped } \\
\text { reads }\end{array}$ & $\begin{array}{l}\text { Average } \\
\text { coverage }\end{array}$ & $\begin{array}{l}\text { Average } \\
\text { coverage } \\
\text { in probes }\end{array}$ \\
\hline N1 & Non-CIMP & F & $68,010,696$ & 2.47 & 61.62 \\
C1 & & & $74,815,980$ & 2.97 & 69.96 \\
N2 & CIMP & M & $62,122,636$ & 2.47 & 63.16 \\
C2 & & & $66,608,688$ & 2.64 & 63.88 \\
N3 & Non-CIMP & M & $57,828,284$ & 2.28 & 57.52 \\
C3 & & & $66,108,442$ & 2.62 & 58.61 \\
N4 & CIMP & M & $59,390,888$ & 2.35 & 61.25 \\
C4 & & $70,070,214$ & 2.56 & 59.0032 \\
N5 & Non-CIMP & M & $68,575,884$ & 2.50 & 49.98 \\
C5 & & & $59,056,548$ & 2.15 & 49.52 \\
N6 & CIMP & F & $79,669,532$ & 2.92 & 71.39 \\
C6 &
\end{tabular}

C CRC, $N$ paired sample of normal mucosa, non-CIMP the mismatch repair gene MLH1 normally expressed, CIMP MLH1 silenced by promoter hypermethylation

a Sample ID changed from arrayexpress

Table 3 Blood data sample characteristics

\begin{tabular}{llll}
\hline Sample ID & Sex & $\begin{array}{l}\text { Number of mapped } \\
\text { reads }\end{array}$ & $\begin{array}{l}\text { Average } \\
\text { coverage }\end{array}$ \\
\hline 1 & M & $390,837,942$ & 12.73 \\
2 & M & $420,368,438$ & 13.70 \\
3 & M & $305,490,164$ & 9.95 \\
4 & F & $383,782,378$ & 12.50 \\
5 & F & $581,667,082$ & 18.86 \\
6 & F & $572,224,352$ & 18.55 \\
\hline
\end{tabular}

a Sample ID changed from source

\section{Blood dataset}

We used data generated by the Blueprint Consortium. We downloaded raw paired-end fastq files from venous blood of 3 healthy females and 3 healthy males (CD14positive, CD16-negative classical monocyte, EGA dataset: EGAD00001002523) (Table 3).

\section{Quality control and mapping}

Quality control was done using fastQC (version 0.11.4) [62]. The reads were subsequently trimmed using TrimGalore! (version 0.4.5) [63]. Reads were mapped to the reference genome using bismark (version 0.18.0). Bowtie2 (version 2.2.9) was used to map to genome hg19 in the CRC dataset, and hg38 in the Blood dataset. Duplicate reads were removed with the deduplicate command from bismark. Deduplicated bam files corresponding to technical replicates in the Blood dataset were merged with samtools merge [64] for each sample. 


\section{SNP calling}

We extracted heterozygous SNPs from the CRC dataset bam files with Bis-SNP (version 1.0.0) [33] by running the BisulfiteGenotyper mode with default parameters, using the dbSNP (Build150) [65] generated VCF file from the NCBI Human Variation Sets (GRCh37p13, last modified:07-10-2017).

\section{methtuple}

methtuple (version 1.5.3) [41] was used to produce a list of unique tuples of size two and the corresponding MM, MU, UM, and UU counts where M stands for "methylated" and U for "unmethylated". The bam files of each sample are those of PE reads and so they were sorted by queryname before using methtuple, as the tool demands it.

\section{Tuple-based ASM score}

We used $\gamma=0.5$ and $\epsilon=0.2$ for all analyses, and allowed for a maximum distance of 150 base pairs between two CpGs in a tuple. Additional file 1: Figure S6 shows

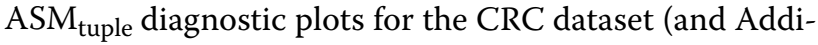
tional file 1: Figure S7 with $\mathrm{ASM}_{\text {snp }}$ ).

\section{ASM $_{\text {tuple }}$ score transformation}

We apply a square root transformation to the $\mathrm{ASM}_{\text {tuple }}$ score before running limma, to get a more stable meanvariance relationship.

$$
L\left(\mathrm{ASM}_{\text {tuple }}\right)=\sqrt{\left|\mathrm{ASM}_{\text {tuple }}\right|}
$$

\section{allelicmeth}

allelicmeth (MethPipe version 3.4.3) [34] is a tool that also detects ASM for a given sample directly from BSseq reads. The tool is part of the MethPipe pipeline [66], which does not use standard bam files. We used commands from the pipeline to transform our bismark bam files from the CRC dataset into $m r$ files, the input to allelicmeth. The output is a bed file with $p$ values for each pair of $\mathrm{CpG}$ sites, reflecting the degree of allele specificity.

\section{amrfinder}

amrfinder (MethPipe version 3.4.3) [34] also detects ASM from the BS-seq reads, however it generates regional scores. As with allelicmeth, we transformed bismark bam files from the CRC dataset into $m r$ files, then ran methstates to generate epiread files, and used these to run amrfinder with default parameters. The output is a bed file with $p$ values for each genomic region with consistent ASM.

\section{Score evaluation}

We converted the $\mathrm{ASM}_{\text {snp }}$ into a tuple-ASM $\mathrm{Anp}_{\text {snp }}$ as $\left|\frac{X_{M}^{i 1 r}+X_{M}^{i 2 r}}{X^{i 1 r}+X^{i 2 r}}-\frac{X_{M}^{i 1 a}+X_{M}^{i 2 a}}{X^{i 1 a}+X^{i 2 a}}\right|$, where 1 and 2 are the the first and second CpG site in a tuple $i$. We treated this converted score as true allele-specific methylation to test our scores at two thresholds: $\geq 0.5$ and $\geq 0.8$.

We transformed the $p$ values generated by allelicmeth and amrfinder with a negative log base 10 . We assigned the same transformed $p$ values to all CpG tuples included in a single amrfinder region.

We also compared to a score based on whether the proportion of methylated reads to total number of reads deviates from 0.5 , but transformed so a value of 0.5 is indicative of high ASM, and 1 or 0 is the lowest ASM. The score is $1-2(\mid$ methylation $-0.5 \mid)$.

We used these four metrics to build ROC curves at different read coverages $(5-9,10-49$ and $\geq 50)$ and at different thresholds of $\mathrm{ASM}_{\mathrm{snp}}$, for a single normal mucosa sample in the CRC dataset.

As an additional validation, we used the Blood dataset to obtain the ASM $\mathrm{Auple}_{\text {te }}$ scores from the promoters of known imprinted genes reported in [11]. Only gene symbols that were traceable with biomaRt $[67,68]$ were included, and genes labelled to be imprinted in placenta were removed, as indicated in $[69,70]$.

\section{t-statistic calculation}

From the limma $R$ package [39], we use lmFit to fit a linear model for each CpG position, and eBayes to calculate a moderated t-statistic on the transformed $\mathrm{ASM}_{\text {tuple }}$ score, or on the $\mathrm{ASM}_{\text {snp }}$ score. For the former, we set the median of two CpGs in a tuple as the CpG position of that tuple. Transformed ASM scores across samples are given as input to lmFit, as well as a design matrix that specifies the conditions of the samples of interest. As specified in $[39,71]$, a CpG site-wise or tuple-wise linear model is defined as

$$
E\left(y_{i}\right)=X \beta_{i}
$$

where for each CpG site or tuple $i$, we have a vector of ASM scores $y_{i}$ and a design matrix $X$ that relates these values to some coefficients of interest $\beta_{i}$.

In the end, we test for a specific contrast that Ho : $C_{\beta_{i j}}=0$.

\section{Smoothing}

We group the positions into genomic clusters using the clusterMaker function from the bumphunter $\mathrm{R}$ package [40]. Then, we use the loessByCluster function to perform loess within each cluster, and obtain $\tilde{\beta}_{i j}$, our smoothed estimate. 


\section{FDR control evaluation}

We selected 6 samples of normal tissue from the CRC dataset and calculated their ASM $_{\text {snp }}$ scores as a baseline in the simulation. We divided the samples in 2 groups of 3 . We generated 1038 clusters of CpGs with the clusterMaker function from the bumphunter package, and set a maximum distance between CpGs of $100 \mathrm{bp}$ (Additional file 1: Figure S8). We chose $20 \%$ of all clusters to be truly differential, and to each of them added effect to a number of randomly selected consecutive CpGs. The effect size is the same for every chosen CpG per cluster, and is obtained by inverse transform sampling of the form $F_{X}^{-1}(u)=x$, where $u \sim \operatorname{Unif}(0.35,0.75)$, and $F_{X}(x)$ the CDF of $\operatorname{Beta}(1,2.5)$ [47] (Additional file 1: Figure S9). In addition, for each truly differential cluster, we randomly selected the sign of the effect size (positive or negative), as well as the group of samples that contains the effect size.

We generated 50 of these simulations, and for each of them, ran DAMEfinder with the cluster-wise correction, and the permutation correction (Additional file 1: Figure S10 for distributions of null and observed areas) with three different $K$ thresholds: $0.2,0.5,0.8$. We used the iCOBRA R package (version 1.12.1) [72] to calculate TPR and FDR at different adjusted $p$ value thresholds: $0.01,0.05,0.1$.

\section{DMR detection}

We identified DMRs with the dmrseq R package (version 1.5.11) [47] for each cancer subtype. We specified the tissue via the testCovariate parameter (CIMP, non-CIMP or normal), and the patient with the adjustCovariate parameter. The cutoff parameter (cutoff of the single CpG coefficient that is used to discover candidate regions) was set as 0.05 and the rest of parameters were set as default.

\section{Supplementary information}

Supplementary information accompanies this paper at https://doi. org/10.1186/s13072-020-00346-8.

Additional file 1. Additional figures.

\section{Acknowledgements}

The authors thank Abdullah Kahraman for technical support at previous stages of the project; Pierre-Luc Germain and Izaskun Mallona for feedback on the manuscript; and the Robinson lab for feedback on figures and analysis.

\section{Authors' contributions}

MM and MDR conceived the study. SO, DM and MDR wrote package, performed analyses. SO, GM and MDR wrote the paper. All authors read and approved the final manuscript.

\section{Funding}

GM and SO acknowledge funding from the SNF Grant 310030-160163/1. MDR acknowledges support from the University Research Priority Program Evolution in Action at the University of Zurich.

\section{Ethics approval and consent to participate}

The bulk of the data used in this study is from public repositories. This study makes use of data generated by the Blueprint Consortium. A full list of the investigators who contributed to the generation of the data is available from http://www.blueprint-epigenome.eu. Funding for the project was provided by the European Union's Seventh Framework Programme (FP7/2007-2013) under grant agreement no 282510 - BLUEPRINT.

\section{Consent for publication}

All authors consent to publication.

\section{Competing interests}

The authors declare that they have no competing interests.

\section{Author details}

${ }^{1}$ Institute of Molecular Life Sciences and SIB Swiss Institute of Bioinformatics, University of Zurich, Winterthurerstrasse 190, 8057 Zurich, Switzerland. ${ }^{2}$ Institute of Molecular Cancer Research, University of Zurich, Winterthurerstrasse 190, 8057 Zurich, Switzerland. ${ }^{3}$ Friedrich Miescher Institute for Biomedical Research, Maulbeerstrasse 66, 4058 Basel, Switzerland.

Received: 9 October 2019 Accepted: 21 May 2020

Published online: 01 June 2020

\section{References}

1. Cedar H, Bergman Y. Linking DNA methylation and histone modification: patterns and paradigms. Nat Rev Genet. 2009;10:295-304. https://doi. org/10.1038/nrg2540.

2. Bonasio R, Tu S, Reinberg D. Molecular signals of epigenetic states. Science. 2010;330(6004):612-6. https://doi.org/10.1126/science.1191078.

3. Bird AP. Use of restriction enzymes to study eukaryotic DNA methylation: II. the symmetry of methylated sites supports semi-conservative copying of the methylation pattern. J Mol Biol. 1978;118(1):49-60. https://doi. org/10.1016/0022-2836(78)90243-7.

4. Suzuki MM, Bird A. DNA methylation landscapes: provocative insights from epigenomics. Nat Rev Genet. 2008;9:465-76. https://doi. org/10.1038/nrg2341.

5. Bergman Y, Cedar H. DNA methylation dynamics in health and disease. Nat Struct Mol Biol. 2013;20:274-81. https://doi.org/10.1038/nsmb.2518.

6. Clark SJ, Statham A, Stirzaker C, Molloy PL, Frommer M. DNA methylation: bisulphite modification and analysis. Nat Protocols. 2006;1(5):2353-64. https://doi.org/10.1038/nprot.2006.324.

7. Liu Y, Siejka-Zielinska P, Velikova G, Bi Y, Yuan F, Tomkova M, et al. Bisulfitefree direct detection of 5-methylcytosine and 5-hydroxymethylcytosine at base resolution. Nat Biotechnol. 2019;37(4):424-9. https://doi. org/10.1038/s41587-019-0041-2.

8. Reddington JP, Pennings S, Meehan RR. Non-canonical functions of the DNA methylome in gene regulation. Biochem J. 2013;451(1):13-23. https ://doi.org/10.1042/BJ20121585.

9. Ferguson-Smith AC. Genomic imprinting: the emergence of an epigenetic paradigm. Nat Rev Genet. 2011;12:565-75. https://doi.org/10.1038/ nrg3032.

10. Bartolomei MS, Ferguson-Smith AC. Mammalian genomic imprinting. Cold Spring Harb Perspect Biol. 2011;3(7). https://doi.org/10.1101/cshpe rspect.a002592.

11. Tucci V, Isles AR, Kelsey G, Ferguson-Smith AC. The Erice Imprinting Group: Genomic imprinting and physiological processes in mammals. Cell. 2019;176(5):952-65. https://doi.org/10.1016/j.cell.2019.01.043.

12. Knoll JHM, Nicholls RD, Magenis RE, Graham JM Jr, Lalande M, Latt SA, et al. Angelman and Prader-Willi syndromes share a common chromosome 15 deletion but differ in parental origin of the deletion. Am J Med Genet. 1989;32(2):285-90. https://doi.org/10.1002/ajmg.1320320235. 
13. Cui H, Onyango P, Brandenburg S, Wu Y, Hsieh C-L, Feinberg AP. Loss of imprinting in colorectal cancer linked to hypomethylation of $\mathrm{H} 19$ and IGF2. Cancer Res. 2002;62(22):6442-6.

14. Schwienbacher C, Gramantieri L, Scelfo R, Veronese A, Calin GA, Bolondi $L$, et al. Gain of imprinting at chromosome 11p15: a pathogenetic mechanism identified in human hepatocarcinomas. Proc Natl Acad Sci. 2000;97(10):5445-9. https://doi.org/10.1073/pnas.090087497.

15. Lyon MF. Gene action in the X-chromosome of the Mouse (Mus musculus L.). Nature. 1961;190(4773):372-3. https://doi.org/10.1038/190372a0.

16. Kerkel K, Spadola A, Yuan E, Kosek J, Jiang L, Hod E, et al. Genomic surveys by methylation-sensitive SNP analysis identify sequence-dependent allele-specific DNA methylation. Nat Genet. 2008;40:904-8. https://doi. org/10.1038/ng.174.

17. Schalkwyk LC, Meaburn EL, Smith R, Dempster EL, Jeffries AR, Davies $M N$, et al. Allelic skewing of DNA methylation is widespread across the genome. Am J Hum Genet. 2010;86(2):196-212. https://doi.org/10.1016/j. ajhg.2010.01.014.

18. Tycko B. Allele-specific DNA methylation: beyond imprinting. Hum Mol Genet. 2010;19(R2):210-20. https://doi.org/10.1093/hmg/ddq376.

19. Gertz J, Varley KE, Reddy TE, Bowling KM, Pauli F, Parker SL, et al. Analysis of DNA methylation in a three-generation family reveals widespread genetic influence on epigenetic regulation. PLoS Genet. 2011;7(8):1-10. https://doi.org/10.1371/journal.pgen.1002228.

20. Onuchic V, Lurie E, Carrero I, Pawliczek P, Patel RY, Rozowsky J, et al. Allelespecific epigenome maps reveal sequence-dependent stochastic switching at regulatory loci. Science. 2018;361(6409). https://doi.org/10.1126/ science.aar3146.

21. Wang H, Lou D, Wang Z. Crosstalk of genetic variants, allele-specific DNA methylation, and environmental factors for complex disease risk. Front Genet. 2019;9:695. https://doi.org/10.3389/fgene.2018.00695.

22. Do C, Lang CF, Lin J, Darbary H, Krupska I, Gaba A, et al. Mechanisms and disease associations of haplotype-dependent allele-specific DNA methylation. Am J Hum Genet. 2016;98(5):934-55. https://doi.org/10.1016/j. ajhg.2016.03.027.

23. Marzi SJ, Meaburn EL, Dempster EL, Lunnon K, Paya-Cano JL, Smith RG, et al. Tissue-specific patterns of allelically-skewed DNA methylation. Epigenetics. 2016;11(1):24-35. https://doi.org/10.1080/15592294.2015.11274 79.

24. Faraday $N$, Yanek LR, Yang XP, Mathias R, Herrera-Galeano JE, Suktitipat $B$, et al. Identification of a specific intronic PEAR1 gene variant associated with greater platelet aggregability and protein expression. Blood. 2011:118(12):3367-75. https://doi.org/10.1182/blood-2010-11-320788.

25. Binder EB, Salyakina D, Lichtner P, Wochnik GM, Ising M, Pütz B, Papiol S, et al. Polymorphisms in FKBP5 are associated with increased recurrence of depressive episodes and rapid response to antidepressant treatment. Nat Genet. 2004;36(12):1319-25. https://doi.org/10.1038/ng1479.

26. Klengel T, Mehta D, Anacker C, Rex-Haffner M, Pruessner JC, Pariante CM, et al. Allele-specific FKBP5 DNA demethylation mediates genechildhood trauma interactions. Nat Neurosci. 2012;16:33-41. https://doi. org/10.1038/nn.3275.

27. Zhang Y, Rohde C, Reinhardt R, Voelcker-Rehage C, Jeltsch A. Non-imprinted allele-specific DNA methylation on human autosomes. Genome Biol. 2009;10(12):138. https://doi.org/10.1186/ gb-2009-10-12-r138.

28. Serre D, Lee $B H$, Ting AH. MBD-isolated Genome Sequencing provides a high-throughput and comprehensive survey of DNA methylation in the human genome. Nucl Acids Res. 2009;38(2):391-9. https://doi. org/10.1093/nar/gkp992.

29. Down TA, Rakyan VK, Turner DJ, Flicek P, Li H, Kulesha E, et al. A bayesian deconvolution strategy for immunoprecipitation-based DNA methylome analysis. Nat Biotechnol. 2008;26:779-85.

30. Laird PW. Principles and challenges of genome-wide DNA methylation analysis. Nat Rev Genet. 2010;11:191-203. https://doi.org/10.1038/nrg27 32.

31. Cheung WA, Shao X, Morin A, Siroux V, Kwan T, Ge B, et al. Functional variation in allelic methylomes underscores a strong genetic contribution and reveals novel epigenetic alterations in the human epigenome. Genome Biol. 2017;18(1):50. https://doi.org/10.1186/s13059-017-1173-7.

32. Zhu P, Guo H, Ren Y, Hou Y, Dong J, Li R, et al. Single-cell DNA methylome sequencing of human preimplantation embryos. Nat Genet. 2018;50(1):12-9. https://doi.org/10.1038/s41588-017-0007-6.
33. Liu Y, Siegmund KD, Laird PW, Berman BP. Bis-SNP: combined DNA methylation and SNP calling for bisulfite-seq data. Genome Biol. 2012;13(7):61. https://doi.org/10.1186/gb-2012-13-7-r61.

34. Fang F, Hodges E, Molaro A, Dean M, Hannon GJ, Smith AD. Genomic landscape of human allele-specific DNA methylation. Proc Natl Acad Sci USA. 2012;109(19):7332-7. https://doi.org/10.1073/pnas.1201310109.

35. Martos SN, Li T, Ramos RB, Lou D, Dai H, Xu J-C, et al. Two approaches reveal a new paradigm of 'switchable or genetics-influenced allelespecific DNA methylation' with potential in human disease. Cell Discov. 2017:3:17038.

36. Robinson MD, Kahraman A, Law CW, Lindsay H, Nowicka M, Weber LM, Zhou X. Statistical methods for detecting differentially methylated loci and regions. Front Genet. 2014;5:324. https://doi.org/10.3389/fgene .2014.00324.

37. Shafi A, Mitrea C, Nguyen T, Draghici S. A survey of the approaches for identifying differential methylation using bisulfite sequencing data. Brief Bioinform. 2018;19:737-53. https://doi.org/10.1093/bib/bbx013.

38. R Core Team. R: a language and environment for statistical computing. Vienna: R Foundation for Statistical Computing; 2019. R Foundation for Statistical Computing. https://www.R-project.org/.

39. Ritchie ME, Phipson B, Wu D, Hu Y, Law CW, Shi W, Smyth GK. limma powers differential expression analyses for RNA-sequencing and microarray studies. Nucl Acids Res. 2015;43(7):47. https://doi.org/10.1093/nar/gkv00 7.

40. Jaffe AE, Murakami P, Lee H, Leek JT, Fallin MD, Feinberg AP, Irizarry RA. Bump hunting to identify differentially methylated regions in epigenetic epidemiology studies. Int J Epidemiol. 2012;41(1):200-9. https://doi. org/10.1093/ije/dyr238

41. Hickey P. Methtuple 2014. https://github.com/PeteHaitch/methtuple.

42. Simes RJ. An improved Bonferroni procedure for multiple tests of significance. Biometrika. 1986;73(3):751-4. https://doi.org/10.1093/biome $\mathrm{t} / 73.3 .751$.

43. Krueger F, Andrews SR. Bismark: a flexible aligner and methylation caller for Bisulfite-Seq applications. Bioinformatics. 2011;27(11):1571-2. https:// doi.org/10.1093/bioinformatics/btr167.

44. Lawrence M, Huber W, Pagès H, Aboyoun P, Carlson M, Gentleman R, et al. Software for computing and annotating genomic ranges. PLoS Comput Biol. 2013;9(8):1-10. https://doi.org/10.1371/journal.pcbi.1003118.

45. Shoemaker R, Deng J, Wang W, Zhang K. Allele-specific methylation is prevalent and is contributed by cpg-snps in the human genome. Genome Res. 2010;20(7):883-9. https://doi.org/10.1101/gr.104695.109.

46. Affinito O, Palumbo D, Fierro A, Cuomo M, Riso GD, Monticelli A, et al. Nucleotide distance influences co-methylation between nearby cpg sites. Genomics. 2019. https://doi.org/10.1016/j.ygeno.2019.05.007.

47. Korthauer K, Chakraborty S, Benjamini Y, Irizarry RA. Detection and accurate false discovery rate control of differentially methylated regions from whole genome bisulfite sequencing. Biostatistics. 2018;007: https://doi. org/10.1093/biostatistics/kxy007.

48. Benjamini Y, Hochberg Y. Controlling the false discovery rate: a practical and powerful approach to multiple testing. J R Stat Soc Ser B. 1995:57(1):289-300.

49. Benjamini Y, Heller R. Screening for partial conjunction hypotheses. Biometrics. 2008;64(4):1215-22. https://doi.org/10.111 1/j.1541-0420.2007.00984.x.

50. Lun ATL, Smyth GK. De novo detection of differentially bound regions for ChIP-seq data using peaks and windows: controlling error rates correctly. Nucl Acids Res. 2014;42(11):95. https://doi.org/10.1093/nar/gku351.

51. Menigatti M, Staiano T, Manser CN, Bauerfeind P, Komljenovic A, Robinson $M D$, et al. Epigenetic silencing of monoallelically methylated miRNA loci in precancerous colorectal lesions. Oncogenesis. 2013;2:56. https://doi. org/10.1038/oncsis.2013.21

52. Parker HR, Orjuela S, Oliveira AM, Cereatti F, Sauter M, Heinrich H, et al. The proto CpG island methylator phenotype of sessile serrated adenomas/polyps. Epigenetics. 2018;13(10-11):1088-105. https://doi. org/10.1080/15592294.2018.1543504.

53. Duncan CG, Grimm SA, Morgan DL, Bushel PR, Bennett BD, Barnabas $B B$, et al. Dosage compensation and DNA methylation landscape of the X chromosome in mouse liver. Sci Rep. 2018;8(1):10138. https://doi. org/10.1038/s41598-018-28356-3. 
54. Carrel L, Willard HF. X-inactivation profile reveals extensive variability in X-linked gene expression in females. Nature. 2005;434(7031):400-4. https ://doi.org/10.1038/nature03479.

55. Wood AJ, Oakey RJ. Genomic imprinting in mammals: emerging themes and established theories. PLoS Genet. 2006;2(11):1-9. https://doi. org/10.1371/journal.pgen.0020147.

56. Hippenmeyer S, Johnson RL, Luo L. Mosaic analysis with double markers reveals cell-type-specific paternal growth dominance. Cell Rep. 2013:3(3):960-7. https://doi.org/10.1016/j.celrep.2013.02.002.

57. Baran Y, Subramaniam M, Biton A, Tukiainen T, Tsang EK, Rivas MA, et al. The landscape of genomic imprinting across diverse adult human tissues. Genome Res. 2015;25(7):927-36. https://doi.org/10.1101/gr.192278.115.

58. Smallwood SA, Lee HJ, Angermueller C, Krueger F, Saadeh H, Peat J, et al. Single-cell genome-wide bisulfite sequencing for assessing epigenetic heterogeneity. Nat Methods. 2014;11:817-20. https://doi.org/10.1038/ nmeth.3035.

59. Farlik M, Sheffield N, Nuzzo A, Datlinger P, Schönegger A, Klughammer $J$, et al. Single-cell DNA methylome sequencing and bioinformatic inference of epigenomic cell-state dynamics. Cell Rep. 2015;10(8):1386-97. https://doi.org/10.1016/j.celrep.2015.02.001.

60. Karemaker ID, Vermeulen M. Single-cell DNA methylation profiling: technologies and biological applications. Trends Biotechnol. 2018;36(9):95265. https://doi.org/10.1016/j.tibtech.2018.04.002.

61. Marusyk A, Polyak K. Tumor heterogeneity: causes and consequences. Biochim Biophys Acta Rev Cancer. 2010;1805(1):105-17. https://doi. org/10.1016/j.bbcan.2009.11.002.

62. Andrews S. fastqc 2015. https://www.bioinformatics.babraham.ac.uk/ projects/fastqc/.

63. Krueger F. Trim Galore! 2017. http://www.bioinformatics.babraham.ac.uk/ projects/trim_galore/.

64. Li H, Handsaker B, Wysoker A, Fennell T, Ruan J, Homer N, et al. The Sequence Alignment/Map format and SAMtools. Bioinformatics. 2009:25(16):2078-9. https://doi.org/10.1093/bioinformatics/btp352.

65. Sherry ST, Ward M-H, Kholodov M, Baker J, Phan L, Smigielski EM, Sirotkin K. dbSNP: the NCBI database of genetic variation. Nucl Acids Res. 2001;29(1):308-11. https://doi.org/10.1093/nar/29.1.308.
66. Song Q, Decato B, Hong EE, Zhou M, Fang F, Qu J, et al. A reference methylome database and analysis pipeline to facilitate integrative and comparative epigenomics. PLoS ONE. 2013;8(12):1-9. https://doi. org/10.1371/journal.pone.0081148.

67. Durinck S, Moreau Y, Kasprzyk A, Davis S, De Moor B, Brazma A, Huber W. BioMart and bioconductor: a powerful link between biological databases and microarray data analysis. Bioinformatics. 2005;21:3439-40. https://doi. org/10.1093/bioinformatics/bti525.

68. Durinck S, Spellman PT, Birney E, Huber W. Mapping identifiers for the integration of genomic datasets with the R/bioconductor package biomaRt. Nat Protocols. 2009;4:1184-91. https://doi.org/10.1038/nprot .2009.97.

69. Court F, Tayama C, Romanelli V, Martin-Trujillo A, Iglesias-Platas I, Okamura $\mathrm{K}$, et al. Genome-wide parent-of-origin DNA methylation analysis reveals the intricacies of human imprinting and suggests a germline methylation-independent mechanism of establishment. Genome Res. 2014;24(4):554-69. https://doi.org/10.1101/gr.164913.113.

70. Pervjakova N, Kasela S, Morris AP, Kals M, Metspalu A, Lindgren CM, et al. Imprinted genes and imprinting control regions show predominant intermediate methylation in adult somatic tissues. Epigenomics. 2016;8(6):789-99. https://doi.org/10.2217/epi.16.8.

71. Smyth GK. Linear models and empirical Bayes methods for assessing differential expression in microarray experiments. Stat Appl Genet Mol Biol. 2004;3(1):1-25. https://doi.org/10.2202/1544-6115.1027.

72. Soneson C, Robinson MD. iCOBRA: open, reproducible, standardized and live method benchmarking. Nat Methods. 2016;13:283. https://doi. org/10.1038/nmeth.3805.

\section{Publisher's Note}

Springer Nature remains neutral with regard to jurisdictional claims in published maps and institutional affiliations.
Ready to submit your research? Choose BMC and benefit from:

- fast, convenient online submission

- thorough peer review by experienced researchers in your field

- rapid publication on acceptance

- support for research data, including large and complex data types

- gold Open Access which fosters wider collaboration and increased citations

- maximum visibility for your research: over 100M website views per year

At BMC, research is always in progress.

Learn more biomedcentral.com/submissions 